\title{
Bar instability in cosmological halos ${ }^{\star}$
}

\author{
A. Curir ${ }^{1}$, P. Mazzei ${ }^{2}$, and G. Murante ${ }^{1}$ \\ 1 INAF - Osservatorio Astronomico di Torino. Strada Osservatorio 20, 10025 Pino Torinese (Torino), Italy \\ e-mail: curir@to.astro.it \\ 2 INAF - Osservatorio Astronomico di Padova. Vicolo Osservatorio 5, 35122 Padova, Italy
}

Received 12 May 2005 / Accepted 30 September 2005

\begin{abstract}
Aims. We investigate the growth of bar instability in stellar disks embedded in a dark matter halo evolving in a fully consistent cosmological framework.

Methods. We perform seven cosmological simulations to emphasise the role of both the disk-to-halo mass ratio and of the Toomre parameter, $\mathrm{Q}$, on the evolution of the disk. We also compare our fully cosmological cases with corresponding isolated simulations where the same halo is extracted from the cosmological scenario and evolved in physical coordinates.

Results. A long living bar, lasting about $10 \mathrm{Gyr}$, appears in all our simulations. In particular, disks expected to be stable according to classical criteria form weak bars. We argue that such a result is due to the dynamical properties of our cosmological halo which is far from stability and isotropy, typical of the classical halos used in the literature; it is dynamically active, has substructures and undergoes infall.

Conclusions. At least for mild self-gravitating disks, the study of the bar instability using isolated isotropic halos in gravitational equilibrium can lead to misleading results. Furthermore, the cosmological framework is needed to quantitatively investigate such an instability.
\end{abstract}

Key words. galaxies: evolution - galaxies: kinematics and dynamics - galaxies: spirals - galaxies: halos

\section{Introduction}

Several works on bar instability in stellar disks have been reported using $N$-body spherical halos (Sellwood 1981; Athanassoula et al. 1987; Debattista \& Sellwood 2000; Patsis \& Athanassoula 2000; Mayer \& Wadsley 2004).

Curir \& Mazzei (1999) were the first to emphasise the role of both the geometry and the dynamical state of a live dark matter (DM) halo in enhancing the bar formation. Progressive efforts to improve models of the halo have been made (Mazzei \& Curir 2001; Athanassoula \& Misiriotis 2002), taking into account the information coming from the cosmological hierarchical clustering scenario of structure formation about density distribution and concentration of DM halos. In the meanwhile, the ever-growing available computing power has made it possible to start simulations of formation and evolution of galaxies in a fully cosmological context. The first works devoted to disk galaxies in such a scenario (Governato et al. 2004; Abadi et al. 2003) have shown that is very difficult to obtain pure disk galaxies mainly because of the high angular momentum loss of the gaseous component. Even with a careful choice of the hosting DM halo, the simulated galaxies appear to have overmassive bulges compared to their disks. Robertson et al. (2004) claim to have overcome most problems, however the bar

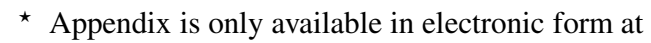
http://www.edpsciences.org instability has not yet been analysed in a cosmological framework. Furthermore, the high CPU cost of such simulations does not yet allow us to explore the role of several parameters related to the phenomenological treatment of the star formation rate and feedback, and the morphologies of the generated galaxies (Mazzei \& Curir 2003; Mazzei 2003).

In this work we present the first attempt to analyse the growth of bar instability in a fully consistent cosmological scenario. We embed a pure stellar disk inside a cosmological halo selected in a suitable slice of the Universe and follow its evolution inside a cosmological framework. We explore how the bar instability behaves and the role of such a scenario. In particular we want to address, besides the role played by the disk-to-halo mass ratio, that of the dynamical state of such halo as given by its substructure and infall, or more generally by its evolution. Our model cannot be viewed as a general, "all-purpose" galaxy evolution model, since the gradual formation and growth of the stellar disk is a fundamental component of the galaxy evolution itself. However our approach allows us to vary parameters like the disk-to-halo mass ratio and the disk temperature, as given by the $Q$ parameter, to analyse the growth of the bar instability and its dependence on such parameters for the first time in a self-consistent cosmological framework. We analyse further the influence of the cosmological environment by comparing these results with those in an isolated scenario with the same halo. 
The plan of the paper is the following: Sects. 2 and 3 describe technical details, in particular the recipe for the initial disk + halo system and our framework, focusing on the cosmological evolution and on the properties of the halo. In Sect. 4 we present the whole set of our disk+halo simulations; in Sect. 5 we describe our results in the cosmological context and the comparison with isolated runs. In Sect. 6 we give our discussion and in Sect. 7 our conclusions. In the Appendix we analyse the robustness of our results, checking for particle resolution and softening length effects.

\section{Numerical method}

Our galaxy model consists of a truncated exponential disk (Curir \& Mazzei 1999), self-consistently embedded in a suitable DM halo extracted from a cosmological simulation. To select the DM halo, we perform a low-resolution $\left(128^{3}\right.$ particles $)$ simulation of a "concordance" $\Lambda \mathrm{CDM}$ cosmological model: $\Omega_{m}=0.3, \Omega_{\Lambda}=0.7, \sigma_{8}=0.9, h=0.7$, where $\Omega_{m}$ is the total matter of the Universe, $\Omega_{\Lambda}$ the cosmological constant, $\sigma_{8}$ the normalisation of the power spectrum, and $h$ the value of the Hubble constant in units of $100 \mathrm{~h}^{-1} \mathrm{~km} \mathrm{~s}^{-1} \mathrm{Mpc}^{-1}$. The box size of our simulation is $25 h^{-1} \mathrm{Mpc}$, which allows us an adequate cosmological tidal field and no boundary effects on our disk. The initial redshift is 20 . We employ the parallel N-body treecode GADGET (Springel et al. 2001). Our initial condition code has been adapted from the setup code of ART (Kravtsov et al. 1997; Kravtsov 1999); (courtesy of A. Klypin).

From this simulation we identify the DM halos at $z=0$ in the mass ${ }^{1}$ range $0.5-5 \times 10^{11} h^{-1} M_{\odot}$, with a standard friendsof-friends algorithm. We discard the halos belonging or near overdense regions (see Sect. 3). Then we follow back the simulation and discard those that undergo significant mergers after a redshift of $\sim 5$. We select one suitable DM halo with a mass $M \sim 10^{11} h^{-1} M_{\odot}$ (at $z=0$ ). We resample it with the multimass technique described in Klypin et al. (2001). The particles of the DM halo, and those belonging to a sphere with a radius $4 h^{-1} \mathrm{Mpc}$, are followed to their Lagrangian position and resampled to an equivalent resolution of $1024^{3}$ particles. The total number of DM particles in the high resolution region is 1216512 which corresponds to a DM mass resolution of $1.21 \times 10^{6} h^{-1} M_{\odot}$. The needed high frequency power is added without modifying the low-frequency Fourier phases of the CDM power spectrum in our low resolution run. The high resolution zone is surrounded by three shells with lower and lower resolution, the lowest one including all the remaining (not resampled) particles among the initial $128^{3}$ set.

The size of the initial Lagrangian region is large enough to resolve with high resolution not only the DM halo, but also its accreting sub-halos. The high-resolution DM halo is followed to the redshift $z=0$. We checked that no lower resolution particles (intruders) are ever present at a radius lower than $\sim 2 h^{-1} \mathrm{Mpc}$ from its centre. This corresponds to the particle with the minimum gravitational energy.

\footnotetext{
${ }^{1}$ In the following, we will refer to the mass as the virial mass i.e. that enclosed in a sphere with overdensity $\delta=\rho / \rho_{\text {crit }}=178 \cdot \Omega_{m}^{0.44}$ (Navarro et al. 2000).
}

Our approach allows us to account for the cosmological tidal field acting on the DM halo and to accurately follow the evolution of the selected halo in a self-consistent way.

We carried out two sets of simulations embedding the galactic disk in the halo at the redshifts $z=2$ and $z=1$ respectively. The first choice corresponds to $10.24 \mathrm{Gyr}$ down to $z=0$ in our chosen cosmology, the second one to $7.71 \mathrm{Gyr}$.

Details of our model disk are presented elsewhere (e.g. Curir \& Mazzei 1999). Here we summarise the main features of the disk. The spatial distribution of the star particles follows the exponential surface density law: $\rho_{\text {stars }}=\rho_{0} \exp -\left(r / r_{0}\right)$ where $r_{0}$ is the disk scale length, $r_{0}=4 h^{-1} \mathrm{kpc}$, and $\rho_{0}$ is the surface central density. The disk is truncated at five scale lengths with a radius: $R_{\text {disk }}=20 h^{-1} \mathrm{kpc}$. To obtain each disk particle's position according to the assumed density distribution, we used the rejection method (Press et al. 1986).

The vertical coordinate is extracted from a Gaussian distribution with a standard deviation equal to $1 \%$ of the disk radius. Circular velocities are assigned analytically to disk particles accounting for the global (disk+cosmological halo) potential, $\Phi$. The radial velocity dispersion $\sigma_{R}$ is assigned through a Toomre parameter $Q . Q$ is initially constant at all disk radii and is it defined as $Q=\frac{\sigma_{R} K}{3.36 G \Sigma}$, where $\kappa$ is the epicyclic frequency, and $\Sigma$ the surface density of the disk. According to the isothermal sheet approximation, the ratio of radial to vertical dispersion is fixed and constant through the disk, moreover the azimuthal dispersion is linked to the radial dispersion via the epicyclic approximation (Hernquist 1993). The final velocity distributions are Gaussian, with the dispersions given above.

Assigning a constant initial $Q$, we can easily classify our disks on the basis of the initial temperature. We explore two values of $Q: 1.5$, which corresponds to a warm disk, and 0.5 , to a cold disk. The average $Q$ value of stars in the Milky Way is estimated between 1 and 3 (Binney \& Tremaine 1987), however the evolution of such a parameter starting from high $z$ is not known.

Our model of a galaxy is very simplified. Neither gas nor star formation is introduced since we aim to focus on the gravitational effect of the halo on the disk and to have hints on the gravitational feedback of the disk itself on the halo. Moreover, our technique is such that the CPU cost of one simulation, while large, is still much lower than the cost of a galaxy formation simulation like that by Abadi et al. (2003), even if our force and mass resolution are comparable. Thus our work could give insights into self-consistent galaxy formation scenarios.

In the following we summarise the main steps of our approach:

i) the halo is identified at redshift $z=0$;

ii) its particles are tracked back to the selected redshift (i.e. $z=1$ and $z=2$ ), and the minimum of their potential well is calculated;

iii) a sphere of radius $R_{\text {sphere }}=3 R_{\text {disk }}$ is extracted from the high resolution simulation; its bulk velocity and the position of its centre are recorded. $R_{\text {sphere }}$ is chosen to ease the comparison of our results with previous numerical work on disk stability, e.g. Curir \& Mazzei (1999) and Mazzei \& Curir (2001). Note that $R_{\text {sphere }} \geq R_{\text {vir }}$ and $M_{\text {halo }}^{\text {vir }} \geq M_{\text {halo }}^{\text {sphere }}$; 
iv) the vector angular momentum $\vec{J}$ and the gravitational potential $\Phi$ are calculated, inside $R_{\text {sphere }}$

v) the disk, in gravitational equilibrium with the potential $\Phi$ and rotating in a plane perpendicular to $\vec{J}$, is generated;

vi) we embed the disk in the high resolution simulation, at the chosen redshift, with its centre of mass in the minimum potential well of the DM halo;

vii) the bulk velocity of the halo is added to the star particles. The cosmological simulation is evolved then, in comoving coordinates, to the final redshift, $z=0$.

\section{The DM halo}

After selecting the halo and resampling at the higher resolution the corresponding Lagrangian region, we run the DM simulation, to extract the halo properties in absence of any embedded stellar disk. The mass of our halo at $z=0,1.03 \times 10^{11} h^{-1} M_{\odot}$, corresponds to a radius, $R_{\mathrm{vir}}=94.7 h^{-1} \mathrm{kpc}$, which involves 84720 halo particles. The nearest DM halo ${ }^{2}$ more massive than $10^{10} h^{-1} M_{\odot}$ is $\sim 1900 h^{-1} \mathrm{kpc}$ away from the centre of our halo; the less massive one, with a mass of $4.6 \times 10^{7} h^{-1} M_{\odot}$, is $\sim 215 h^{-1} \mathrm{kpc}$ away. Moreover, the behaviour of the density contrast, $\delta$, is monotonically decreasing with the radius, and $\delta$ falls below unity at $\sim 550 \mathrm{~h}^{-1}, 450 \mathrm{~h}^{-1}, 350 \mathrm{~h}^{-1}$ physical kpc away from the centre of our halo at $z=0, z=1$ and $z=2$ respectively. Therefore, we conclude that the selected halo lies in an under-dense environment.

The accretion history of our halo has been calculated as follows. Starting from redshift $z_{1}=0$, we identified DM halos using the public halo finder SKID $^{3}$ (Stadel 2001) at the redshift $z_{2}=z_{1}+\mathrm{d} z_{1}$, corresponding to our previous simulation output. We then define as progenitor of our halo a SKID group at the redshift $z_{2}$, if at least a fraction $f=30 \%$ of its particles come from the halo at the redshift $z_{1}$. We also identify as accreting field particles all the DM particles not belonging to any SKID group but belonging to the halo. We then iterate the procedure, using the simulation output corresponding to the redshift $z_{3}=$ $z_{2}+\mathrm{d} z_{2}$; the progenitors are now all the groups that have at least a fraction $f$ of particles coming from progenitors at $z_{1}$ or from the accreting field particles, and so on for the earlier redshifts. We check that the qualitative behaviour of the accretion history is not dependent on the value of $f$ (we also tested $f=20 \%$ and $f=50 \%$ ) and on the parameters used in SKID (we use a typical object size $\tau=5 h^{-1} \mathrm{kpc}$ but also the effect of $3 h^{-1} \mathrm{kpc}$ and $6 h^{-1} \mathrm{kpc}$ have been explored). From Fig. 1, we note that the halo suffers its last major merger (i.e. a merger between two progenitors whose masses have a ratio which is not larger than 3 ) at $z=9$. After $z \sim 5$, the most important contribution to its mass comes from accreting field particles. This contribution declines after $z \sim 2$ becoming less important. At $z \sim 0.9$, the total accreting mass is smaller than the mass of the larger subhalo. Thus we conclude that our halo undergoes no significant

\footnotetext{
${ }^{2}$ Halos have been identified using the friends of friends algorithm with a linking length $l=0.15$, mean interparticle distances, with more than 8 particles.

${ }^{3}$ http://www-hpcc.astro.washington.edu/ tools/skid.html
}
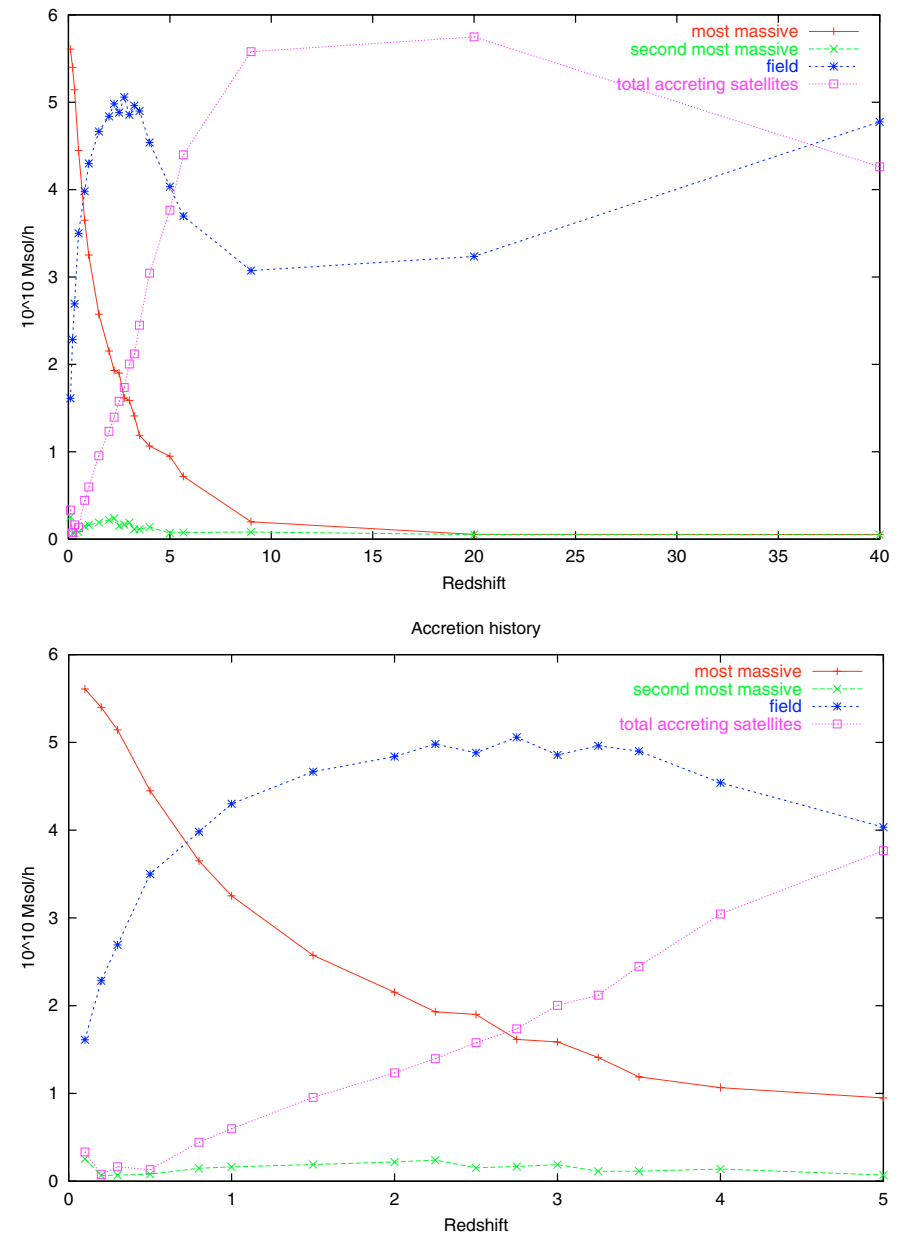

Fig. 1. Accretion history of our selected DM halo as a function of the redshift: top panel from redshift $z=40$ to $z=0$, bottom panel from $z=5$ to $z=0$; solid (red) line shows the mass of the most massive progenitor of the halo, long-dashed (green) line that of the second most massive progenitor, dotted (magenta) line the total mass of progenitors (the most massive excluded), and short-dashed (blue) line the total mass of field particles.

merger during the time it hosts our stellar disk, nor immediately before.

The properties of the selected halo at three relevant redshifts are listen in Table 1. Its density profile is well-fitted by a NFW form (Navarro et al. 1996, 1997) at $z \leq 2$. The concentration, $C_{\text {vir }}{ }^{4}$ here defined as $R_{\text {vir }} / R_{s}$, has a high value, 18.1 , confirming that this halo does "form" at quite high redshift (see Wechsler et al. (e.g. 2002) for a discussion about the link between concentration and assembly history of the halo). The dimensionless spin parameter of the halo is defined as: $\lambda=\frac{J}{\sqrt{2} M V R}$ (Bullock et al. 2001) where $J$ is the angular momentum inside a sphere of radius $R$ and $V$ is the halo circular velocity, $V^{2}=G M / R$. Its values in Table 1 are near to the

4 We note however that $C_{\mathrm{NFW}}$ is defined against $R_{200}$, the radius enclosing a sphere with overdensity equal to 200 times the critical density of the Universe, and not against $R_{\mathrm{vir}}$ as here; therefore, in our cosmological model, it is always $C_{\mathrm{NFW}}<C_{\mathrm{vir}}$. At $z=0$ our halo has $C_{\mathrm{NFW}} \sim 14$. 
Table 1. DM halo properties.

\begin{tabular}{cccccccc}
\hline \hline$z$ & $C_{\mathrm{vir}}$ & $M_{\mathrm{vir}}$ & $R_{\mathrm{vir}}$ & $N_{\text {part }}$ & $\lambda$ & $\tau_{60}$ & $\tau_{20}$ \\
\hline 0 & 18.0 & $1.03 \cdot 10^{11}$ & 94.7 & 84720 & 0.01 & 0.60 & 0.80 \\
1 & 13.2 & $7.7 \cdot 10^{10}$ & 51.6 & 63886 & 0.02 & 0.78 & 0.92 \\
2 & 8.3 & $5.2 \cdot 10^{10}$ & 30.9 & 42660 & 0.04 & 0.92 & 0.90 \\
\hline
\end{tabular}

Column 1: redshift; Col. 2: concentration parameter $C_{\text {vir }}$; Col. 3 : virial mass, in $h^{-1} M_{\odot}$; Col. 4: virial radius, in $h^{-1}$ physical kpc; Col. 5: number of DM particles within the virial radius; Col. 6: spin parameter; Col. 7: triaxiality parameter within a sphere of radius $60 \mathrm{~h}^{-1}$ physical kpc; Col. 8: triaxiality parameter within a sphere of radius $20 h^{-1}$ physical kpc.

average ones for our cosmological model $(\lambda=0.035$; Maller et al. 2002)

\section{Disk simulations}

We performed seven simulations of the disk+halo system as described below (Sect. 4.1). By comparing results of such a set of 7 simulations (Sect. 5) with the DM-only, we separate the effect of the stellar disk on the halo evolution in the cosmological framework. Several simulations of the disk+halo isolated system are also run, to separate the effect of the cosmological environment (Sects. 4.2 and 5.2). We used 56000 star particles to describe our disk; the (Plummer-equivalent) softening length, the same for DM and star particles, is $0.5 h^{-1} \mathrm{kpc}$ in comoving coordinates $^{5}$. We used a time-step criterion based on the local dynamical time (criterion " 3 " of the GADGET code), which provides $2-6 \times 10^{4}$ time-steps from $z=2$ to $z=0$ (except one case, simulation 5 of Table 2. The most CPU-expensive of our simulations needed $\sim 5000 \mathrm{CPU}$ hours to be completed on the SP4 computer (CINECA computing center).

\subsection{Cosmological cases}

The main parameters and the initial properties of this set of simulations are listed in Table 2 .

A global stability criteria for bar instability in a disk galaxy is the one analysed in Efstathiou et al. (1982). The parameters $\alpha r_{m}$ and $\frac{v_{m}}{(\alpha M G)^{1 / 2}}$ (where $v_{m}$ is the maximum value of the disk rotational curve, $r_{m}$ the corresponding radius, $\alpha=r_{0}{ }^{-1}$ and $M$ is the disk mass) have been defined. Efstathiou et al. (1982) stated the criterion $\frac{v_{m}}{(\alpha M G)^{1 / 2}} \geq 1.1$ over the range $0.1 \leq \alpha r_{m} \leq 1.3$ for a disk model being stable to bar formation. The values of these parameters are reported in Table 2.

Simulations c1, c2 and c3 in Table 2 refer to a cold disk $(Q=0.5)$. In simulation $\mathrm{c} 1$, at the final time (i.e. $z=0$ ) the baryon fraction inside $R_{\text {vir }}, f_{\mathrm{b}}=M_{\text {disk }} / M_{\text {disk }+\mathrm{DM}} \sim 0.34$, is $44 \%$ less than its initial value, 0.53 . The final baryon fraction of simulation $\mathrm{c} 2$ is $\simeq 0.16$, compared with its initial value, 0.28 . Simulation $\mathrm{c} 3$ provides $f_{\mathrm{b}} \simeq 0.05$ at $z=0,50 \%$ less than its initial value. Simulations c4 and c5 provide the same

\footnotetext{
${ }^{5}$ Note that, since the disk is modelled in physical coordinates and embedded in the cosmological halo at redshifts $z=2$ and $z=1$, its thickness is larger than the value of the Plummer softening we use.
}

final baryon fractions as the corresponding simulations with the lower Toomre's parameter.

Simulation c6 and c7, which explore the role of the initial redshift on the bar instability, provide similar final values of the baryon's fraction as the corresponding simulations c2 and c3. Neither the Toomre parameter nor the initial redshift affect the evolution of this ratio which is driven by the mass of the stellar disk. While the baryon fraction of simulation $\mathrm{c} 1$ is too high to be consistent with the cosmological value 0.166 (Ettori 2003), all the other simulations give baryon fractions in the allowed range. We however emphasise that the aim of the current work is not to build a realistic galaxy model, but to study the effect of different halo-to-disk mass ratios on the onset of the bar instability. We verify that the inclusion of the disk does not result in significant changes in the accretion history of the DM halo.

\subsection{Isolated cases}

We also performed several simulations of the isolated disk+halo system using the same halo as extracted from our cosmological simulations at $z=2$ (Sect. 5.2 and Appendix). By comparing results of this set of simulations with the previous ones we aim to disentangle the effect of the large scale cosmological structure and of cosmological expansion on the system evolution. Moreover, such results are directly comparable both with our previous works (Curir \& Mazzei 1999; Mazzei $\&$ Curir 2001) and with those in the literature (Athanassoula et al. 1987). The initial and final values for these simulations are listed in Tables 2 and 3. Our isolated halo is produced by a non dissipative collapse in a cosmological scenario. As a consequence its mass distribution is not spherically symmetric. Moreover such a halo is anisotropic and endowed with a spin parameter and substructure. Therefore it is different from the standard isolated halos used in the literature to study bar instabilities, since it keeps a relic cosmological signature. When the halo is extracted from its cosmological environment, the large scale structure, the continuing matter infall and the expansion of the Universe no longer influence its evolution. Such a halo cannot be in gravitational equilibrium, because its evolution is not yet completed either at $z=2$ or at $z=1$, as shown in Fig. 1 . For this reason, the results presented below, concerning the behaviour of the disk embedded in such a "isolated" halo have to be compared with similar cases in which non-equilibrium DM halos are used, as e.g. in Curir \& Mazzei (1999); Mazzei $\&$ Curir (2001). After subtracting the CM velocity and embedding the disk, as described in Sect. 2 items $i-v$, we integrated the system in physical coordinates (the effect of the cosmological expansion is therefore ruled out in these models). A further difference is that the softening length is now in physical units. We have at least 10000 time steps from the initial time to $t=10.24 \mathrm{Gyr}$ corresponding to redshift 0 .

Finally, in order to disentangle the effect of the geometry and of the spin of an isolated halo we also performed two simulations using a Navarro Frenk and White (NFW) halo having the same virial radius and mass as our cosmological one. The initial and final values of these two simulations are listed in the two last lines of Tables 2 and 3. 
Table 2. Simulations: initial values.

\begin{tabular}{ccccccccc}
\hline \hline$N$ & $Q$ & $M_{\text {disk }}$ & $z$ & $M_{\mathrm{DM}}$ & $R_{\mathrm{DM}}$ & $\alpha r_{\mathrm{m}}$ & $\frac{v_{m}}{\left(\alpha G M_{\text {disk }}\right)^{1 / 2}}$ & halo \\
\hline $\mathrm{c} 1$ & 0.5 & 1 & 2 & 0.64 & 0.64 & 1.9 & 0.67 & \\
$\mathrm{c} 2$ & 0.5 & 0.33 & 2 & 0.64 & 1.94 & 1. & 1.08 & \\
$\mathrm{c} 3$ & 0.5 & 0.1 & 2 & 0.64 & 6.4 & 0.9 & 1.68 & \\
c4 & 1.5 & 0.33 & 2 & 0.64 & 1.94 & 1. & 1.08 & \\
c5 & 1.5 & 0.1 & 2 & 0.64 & 6.4 & 0.9 & 1.68 & \\
c6 & 0.5 & 0.33 & 1 & 0.67 & 2.0 & 1.05 & 1.05 & \\
c7 & 0.5 & 0.1 & 1 & 0.67 & 6.7 & 1. & 1.6 & \\
i1 & 1.5 & 0.33 & & 0.64 & 1.94 & 1 & 1.08 & cosm \\
i2 & 1.5 & 0.1 & & 0.64 & 6.4 & 0.9 & 1.68 & cosm \\
i3 & 1.5 & 0.33 & & 0.64 & 1.94 & 1 & 1.08 & cosm/frozen disk \\
i4 & 1.5 & 0.33 & & 0.95 & 2.87 & 0.85 & 1.5 & NFW \\
i5 & 1.5 & 0.1 & & 0.95 & 9.5 & 1.27 & 1.25 & NFW \\
\hline
\end{tabular}

Column 1: simulation number and simulation type (c: cosmological simulations, i: isolated simulations); Col. 2: $Q$ initial value of the disk; Col. 3: mass of the disk in $5.9 \times 10^{10} M_{\odot}$; Col. 4: initial redshift (for the cosmological cases); Col. 5: initial DM mass inside the disk radius; Col. 6: initial halo-to-disk mass ratio inside the disk radius; Cols. 7 and 8: Efstathiou et al. (1982) parameters, where: $\alpha=r_{0}{ }^{-1}, v_{\mathrm{m}}$ is the maximum rotational velocity, and $r_{\mathrm{m}}$ the corresponding radius; Col. 9: type of halo used (for the isolated cases).

Table 3. Simulations: final results.

\begin{tabular}{cccccccc}
\hline \hline$N$ & $M_{\mathrm{DM}}$ & $R_{\mathrm{DM}}$ & $S_{\mathrm{m}}$ & $Q_{\mathrm{t}}$ & $a_{\max }$ & bulge & bars in bars \\
\hline $\mathrm{c} 1$ & 0.79 & 0.8 & 0.42 & 0.38 & 7 & $\mathrm{y}$ & $\mathrm{n}$ \\
$\mathrm{c} 2$ & 0.77 & 2.39 & 0.33 & 0.44 & 8 & $\mathrm{y}$ & $\mathrm{n}$ \\
$\mathrm{c} 3$ & 0.73 & 7.41 & 0.8 & 0.07 & 3.8 & $\mathrm{n}$ & $\mathrm{y}$ \\
$\mathrm{c} 4$ & 0.78 & 2.40 & 0.48 & 0.37 & 5 & weak & $\mathrm{n}$ \\
$\mathrm{c} 5$ & 0.73 & 7.41 & 0.70 & 0.08 & 6.5 & $\mathrm{n}$ & $\mathrm{y}$ \\
$\mathrm{c} 6$ & 0.79 & 2.43 & 0.35 & 0.42 & 6.8 & weak & $\mathrm{n}$ \\
$\mathrm{c} 7$ & 0.77 & 7.73 & 0.58 & 0.16 & 5.0 & $\mathrm{n}$ & $\mathrm{y}$ \\
$\mathrm{i} 1$ & 0.73 & 2.21 & 0.25 & 0.4 & 9.5 & $\mathrm{y}$ & $\mathrm{n}$ \\
$\mathrm{i} 2$ & 0.51 & 5.1 & 0.68 & 0.1 & 8 & $\mathrm{n}$ & $\mathrm{y}$ \\
$\mathrm{i} 3$ & 0.7 & 2.12 & 0.3 & 0.39 & 10 & $\mathrm{y}$ & $\mathrm{n}$ \\
$\mathrm{i} 4$ & 1. & 3.03 & 0.33 & 0.42 & 6 & $\mathrm{y}$ & $\mathrm{n}$ \\
15 & 0.95 & 9.5 & 0 & 0 & 0 & $\mathrm{n}$ & no bar \\
\hline
\end{tabular}

Column 1: simulation number and simulation type; Col. 2: DM mass inside the disk radius in $5.9 \times 10^{10} M_{\odot}$; Col. 3 : halo-to disk mass ratio inside the disk radius; Col. 4: maximum bar strength at $z=0$ : strong bar Mazzei \& Curir (2001) require $S_{\mathrm{m}} \leq 0.6$; Col 5: bar strength evaluated according to Combes \& Sanders (1981), stronger bar corresponds to higher values of $Q_{\mathrm{t}}$; Col. 6: major axis (physical kpc) corresponding to the maximum bar strength; Col. 7: morphology of the inner region of the disk; Col. 8: peculiar features inside the disk.

\section{Results}

In this section we present the evolution of isodensity contours of the different cosmological simulations. From these contours we evaluate the final bar strengths which are reported in Table 3. Spatial resolution of the maps is always $0.5 h^{-1}$ physical $\mathrm{kpc}$ and the box size is 40 times the spatial resolution. Contours are computed at 11 fixed levels ranging from $2 \times 10^{-4}$ to 0.015 in terms of fraction of stars/spatial resolution within the total number-density of stars in the map. Following Curir \& Mazzei (1999), we define, as a measure of the bar strength, the maximum value of the axial ratio, $S_{m}=b / a$ (Table 3 ): a strong bar corresponds to $S_{m} \leq 0.6$ or to an ellipticity, $\epsilon=(1-b / a)$, larger than 0.4 .

\subsection{Morphologies of the stellar disk in the cosmological framework}

Figures 2-4 show the evolution of isodensity contours of simulations c1, c2 and c3. More massive cold disks suffer stronger lopsided instability ( $m=1$ ) from the beginning of their evolution which degenerates in the $m=2$ instability, i.e. the bar instability, later on. The less massive cold disks, i.e. DM dominated cases, show a weaker $m=1$ instability. Thus bar instability develops earlier than in the corresponding more massive cases and the disk attempts to re-arrange before the end of the simulation. Figures 5 and 6 compare face-on, side-on and edge-on isodensity contours of simulations $\mathrm{c} 1$ and $\mathrm{c} 2$ at $z=0$. Our cold intermediate mass case shows peanut-shape in the side-on view and bulge-like contours in the edge-on view. Therefore, in this a case, a bulge could be mis-identified due to the bar feature. However in the warm analogous case (Fig. 7) this feature does not arise. On the other hand, our more massive cold disk shows edge-on isodensity contours with a less defined inner bulge and thicker disk-like contours in the outer regions. Its side-on view corresponds to a boxy image without an extreme peanut feature.

Therefore the halo-to-disk ratio has a significant influence on the stellar disk at $z=0$.

The $Q$ parameter does not influence the final $(z=0)$ morphologies of our less massive disks, always showing disk-like shapes (Fig. 8). This suggests that the cold intermediate mass case is peculiar as far as the peanut shape is concerned. Such a feature has been recognized by Combes \& Sanders (1981) as caused by vertical orbital resonances. Figure 9 shows the isodensity contours of simulation $\mathrm{c} 4$. In this simulation the higher value of the $Q$ parameter stabilises the disk against the local 

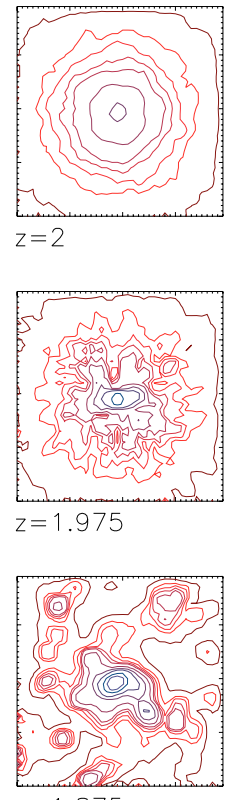

$z=1.875$

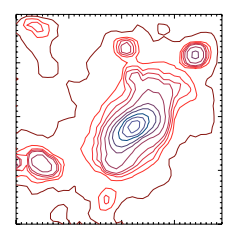

$z=1.75$

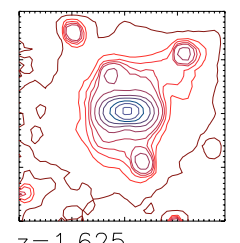

$z=1.625$

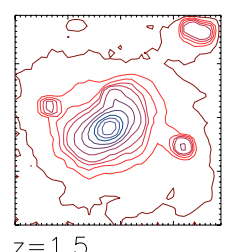

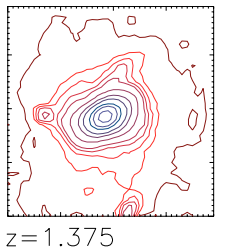

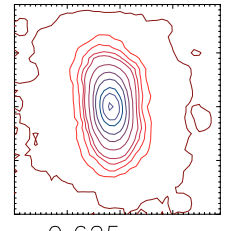

$z=0.625$
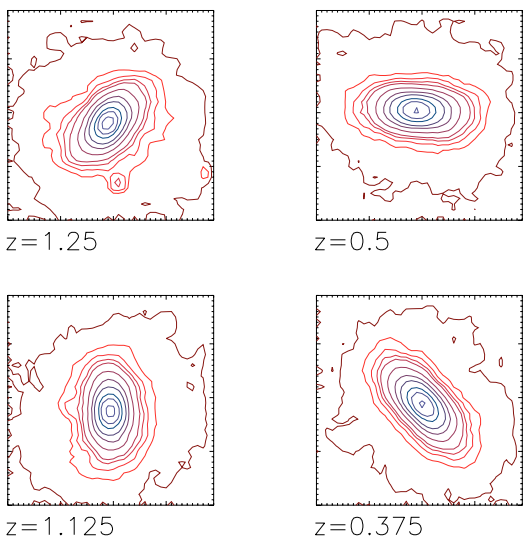

$z=0.375$
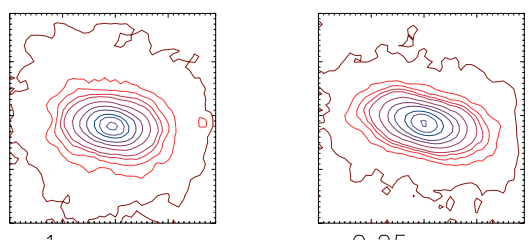

$z=0.25$
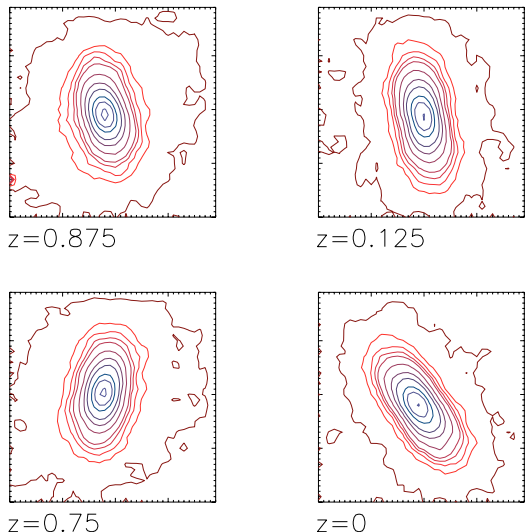

Fig. 2. Evolution of isodensity contours of simulation c1 at 11 fixed levels (see Sect. 5). The size of all the frames is $20 \mathrm{~h}^{-1}$ physical kpc, here and in all the following figures in which isodensity contours are shown.

Jeans instability and the bar appears later than in the corresponding cold case (simulation c2).

Therefore warmer disks are more stable against lopsided instability than the corresponding cold cases. Inside warmer and less massive disks, bars in bars, namely bar features at different isodensity levels, nested with twisting major axes, are also shown.

Isodensity contours of simulation c6 (Fig. 10), are similar to those of the corresponding simulation $\mathrm{c} 2$ (Fig. 6), which however starts at $z=2$. Morphologies of both simulations c6 and c7 show thinner disks than simulations $\mathrm{c} 4$ and c5
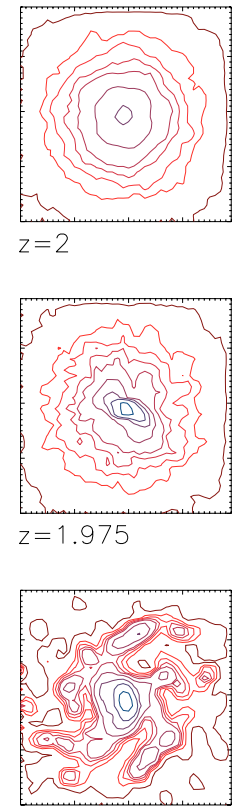

$z=1.875$

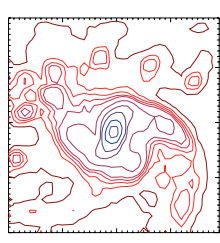

$z=1.75$
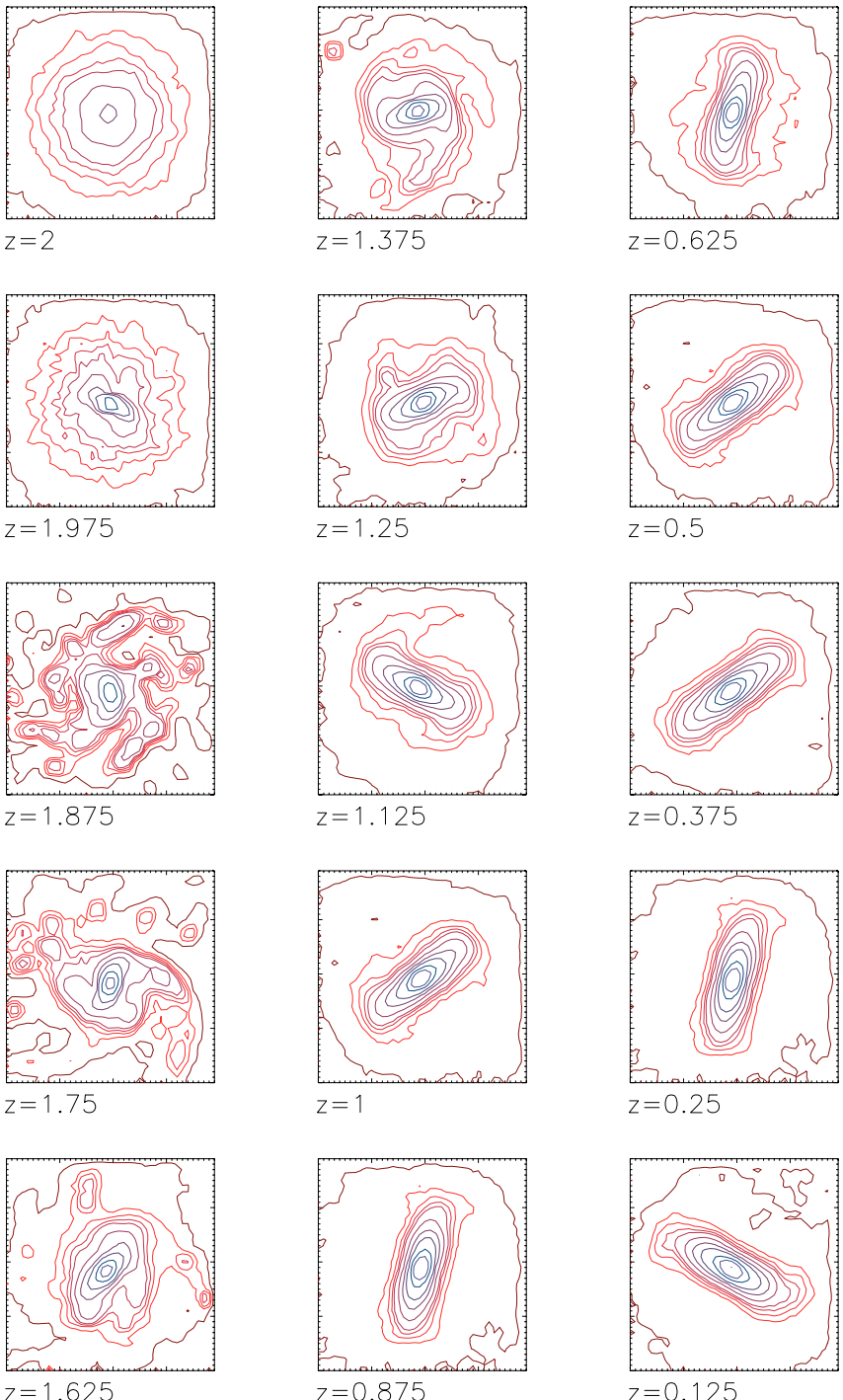

$z=1.25$
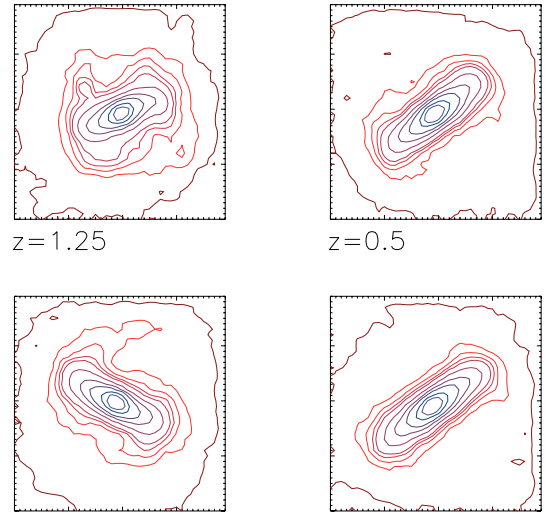

$z=1.125$

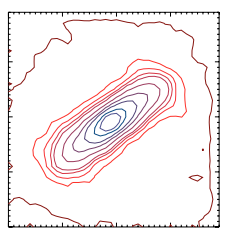

$z=0.375$
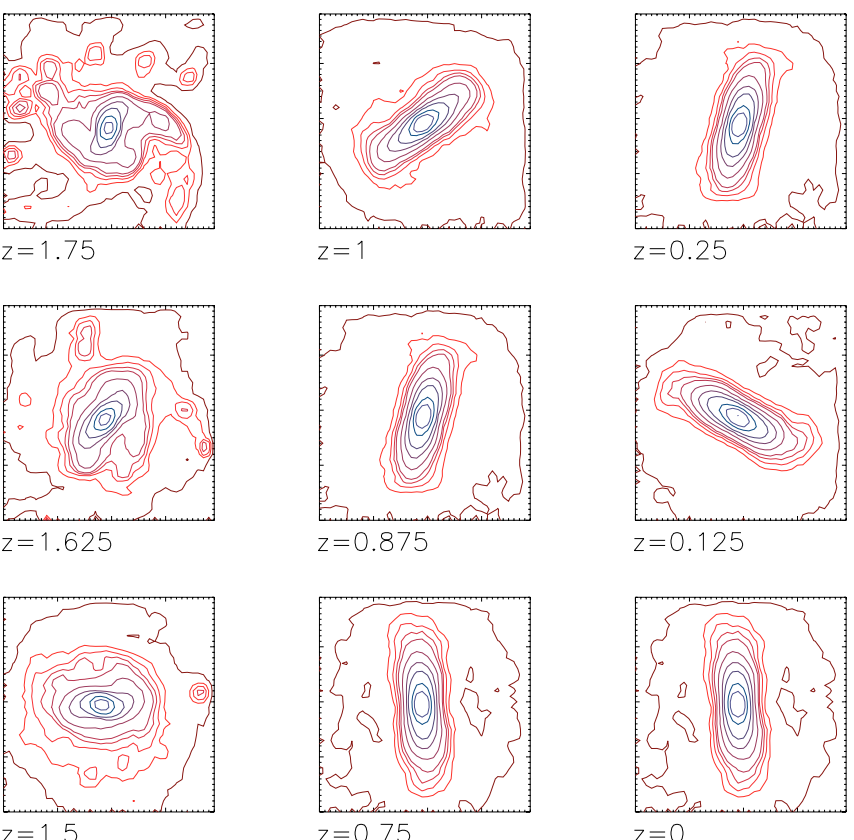

Fig. 3. Evolution of density contours of simulation c 2 as described in Fig. 2.

given their shorter evolutionary time $(\approx 7.7$ Gyr instead of $\approx 10.24$ Gyr).

\subsubsection{The bar strength}

A variety of quantitative parameters have been suggested to evaluate the strength of the bar (see Buta \& Block 2001, for a review). First we quantify the growth of the bar instability studying the time evolution of the ellipticity of our isodensity contours as a function of their major axis, $a$. The strength of the bar depends on the density contrast accounted for, and it varies 

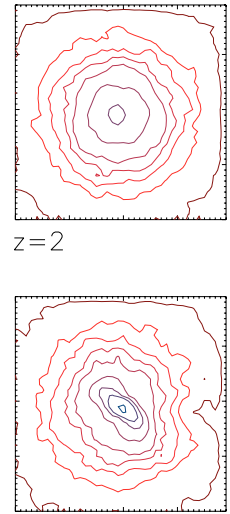

$z=1.975$

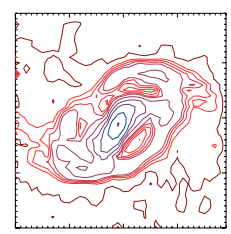

$z=1.875$

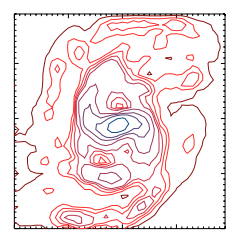

$z=1.75$

$z=1.625$

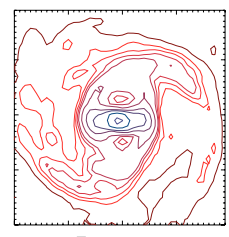

$z=1.5$

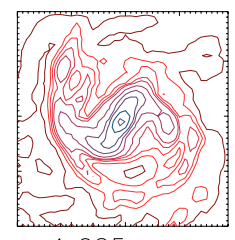

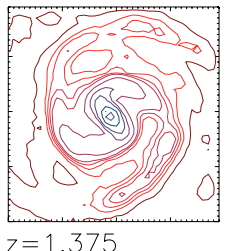
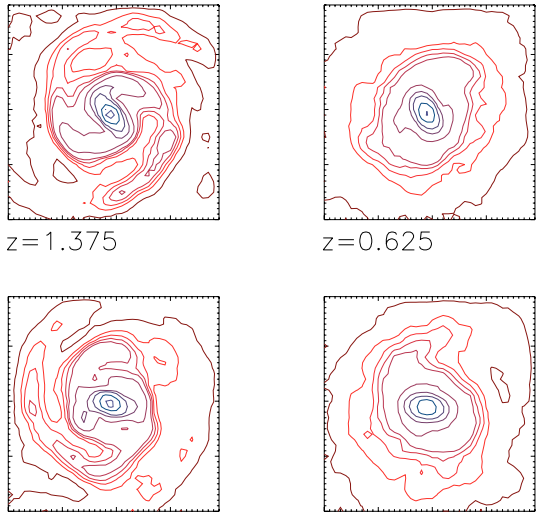

$z=1.25$

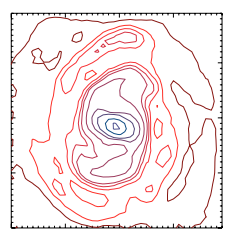

$z=1.125$

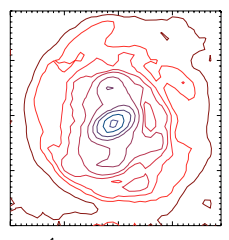

$z=1$

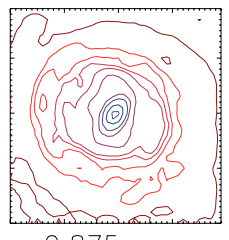

$z=0.875$

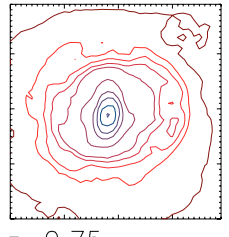

$z=0.75$
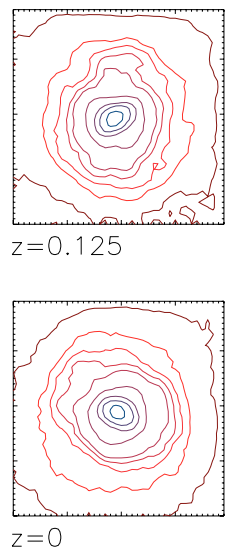
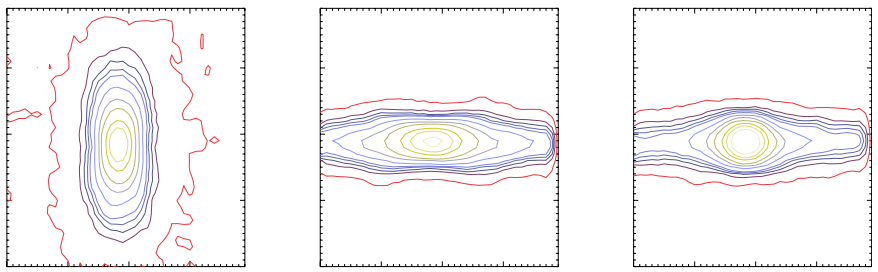

Fig. 5. Face-on, side-on and edge-on view of isodensity contours of simulation $\mathrm{c} 1$ at $z=0$.
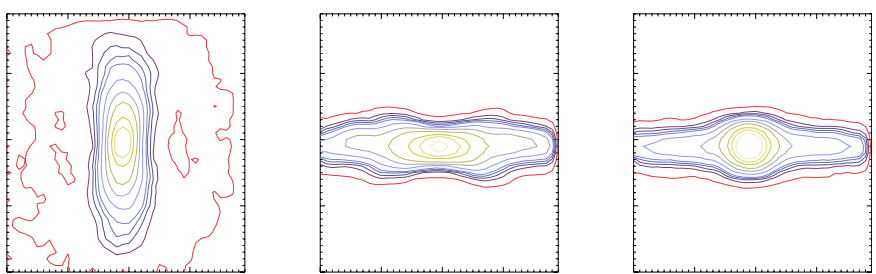

Fig. 6. Face-on, side-on and edge-on view of isodensity contours of simulation $\mathrm{c} 2$ at $z=0$.
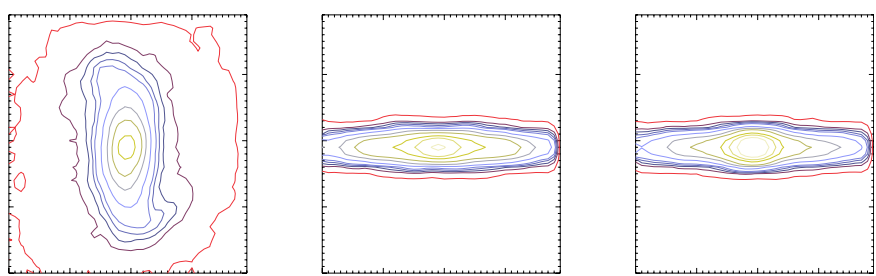

Fig. 7. Face-on, side-on and edge-on isodensity contours at $z=0$ of simulation $\mathrm{c} 4$ in Table 2.

Fig. 4. Evolution of isodensity contours of simulation c3 as described in Fig. 2.

with the distance from the centre; different choices can change its value but not the trend outlined in Table 3 .

Figure 12 shows that in simulation $\mathrm{c} 1$ the strength of the bar increases with time. The length of the bar depends on the redshift too: it grows until $z=0.5$, and then shrinks to $z=0$.

By comparing Figs. 13 and 14, which show the ellipticity profiles of simulations $\mathrm{c} 2$ and $\mathrm{c} 4$ respectively, we point out that a greater $Q$, in intermediate mass disks, directly affects the bar strength: a stronger local gravitational instability, corresponding to a lower $Q$ value, triggers a stronger bar (Table 3 ). For the less massive disks, $Q$ poorly influences the bar strength. In these cases, the local Jeans instability has a small impact on the
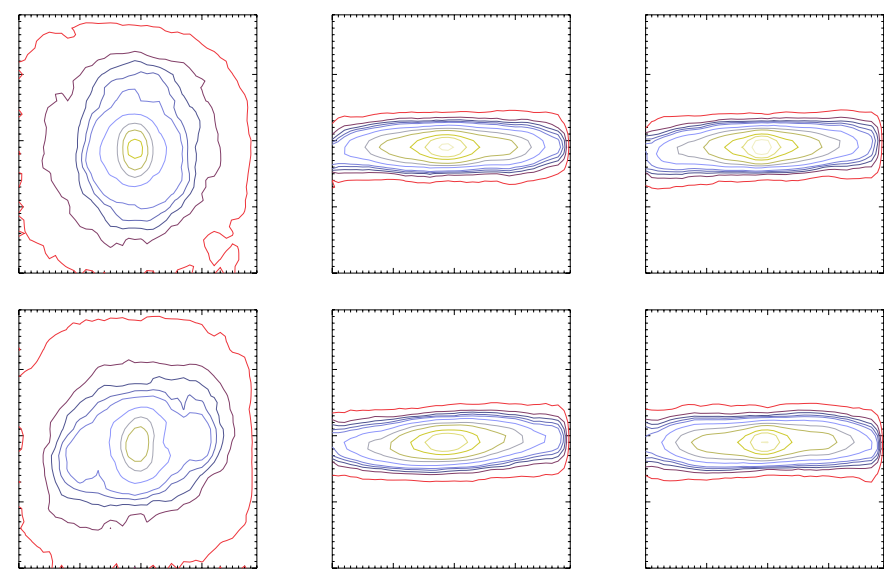

Fig. 8. Face-on, side-on and edge-on isodensity contours at $z=0$ of simulation c3 (top panels) and c5 (bottom panels) in Table 2.

bar formation and evolution, which is dominated instead by the dynamics of the DM halo.

The embedding redshift does not have a major impact on the bar strength. Its more important effect is the change of the bar length which can be related to the larger time span of simulation c2 (or c3) with respect to simulation c6 (or c7). Therefore, the halo evolution between $z=2$ and $z=1$ does not seriously affect the disk instability, at least for the cold disk cases. 

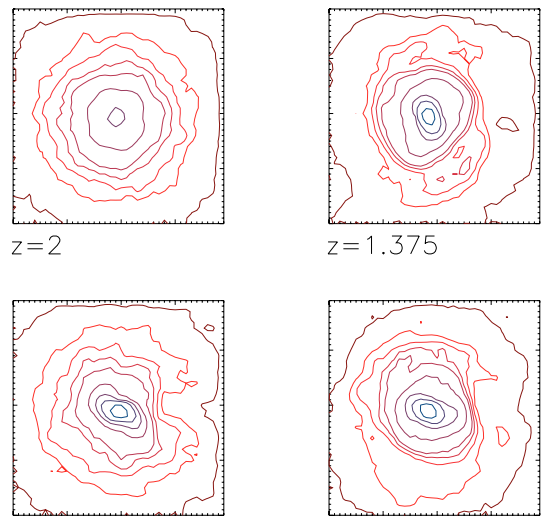

$z=1.975$

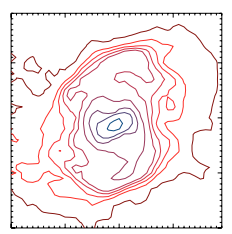

$z=1.875$
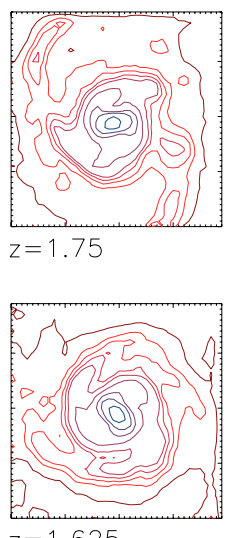

$z=1.625$

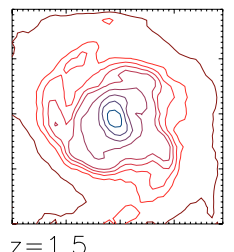

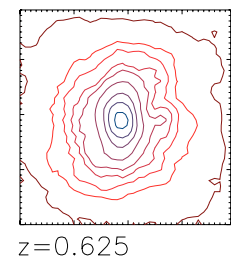

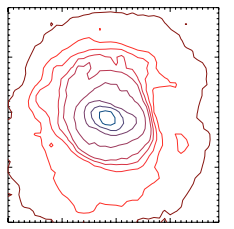

$z=1.25$
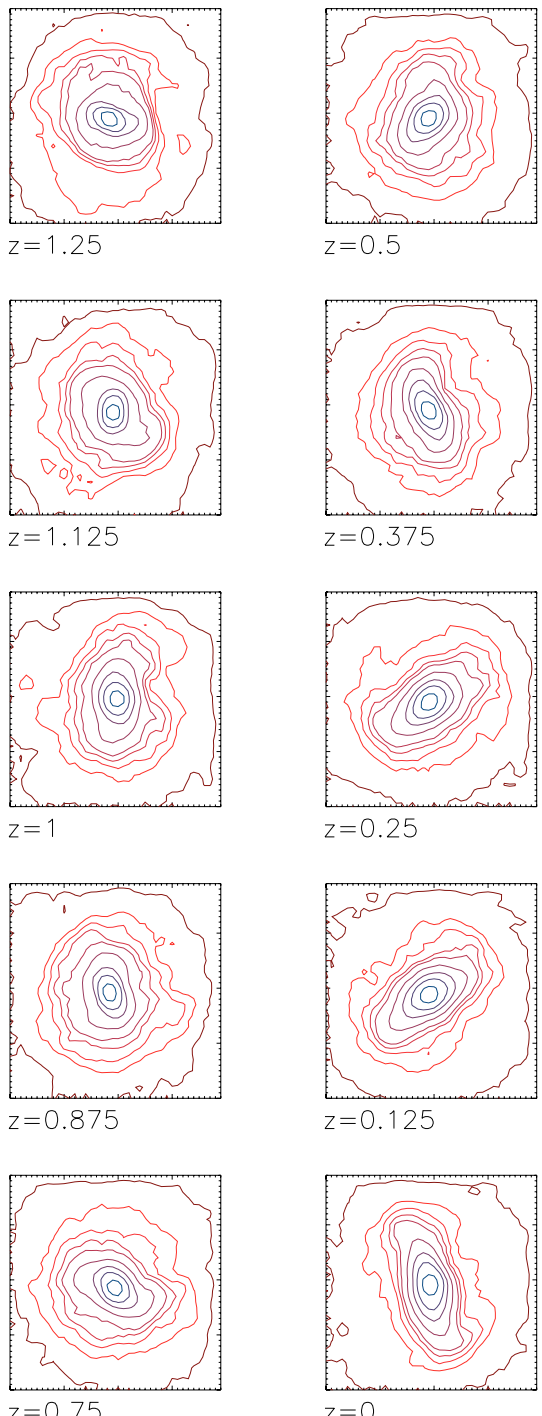

$z=0.375$

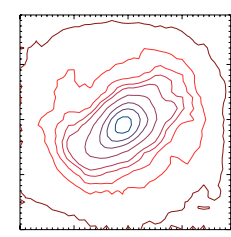

$z=0.25$

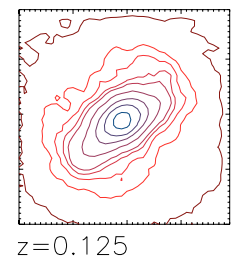

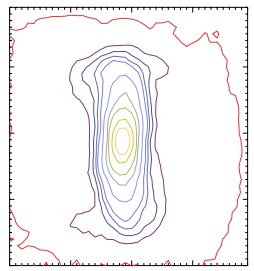
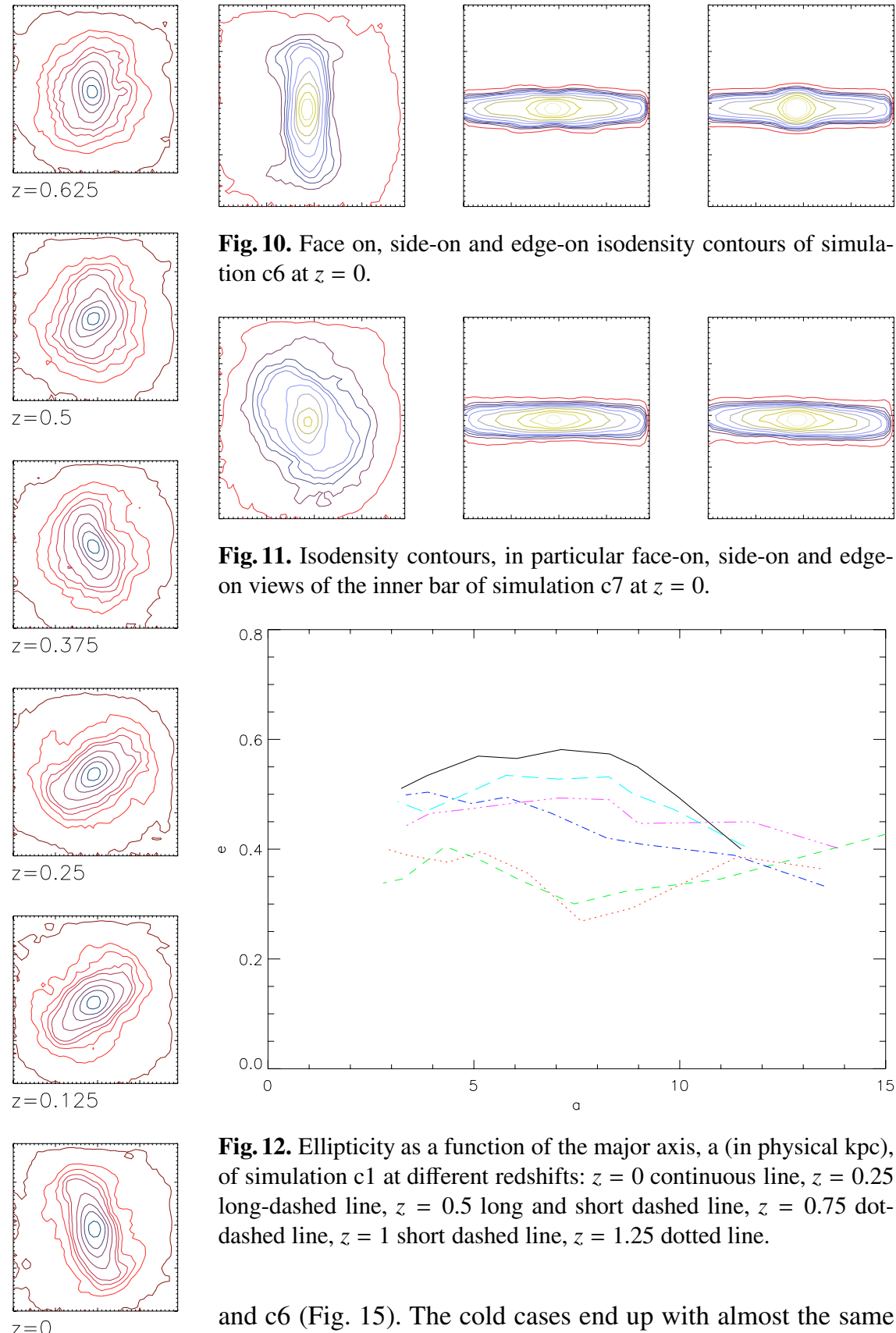

$\mathrm{z}=0$

Fig. 10. Face on, side-on and edge-on isodensity contours of simulation $\mathrm{c} 6$ at $z=0$.
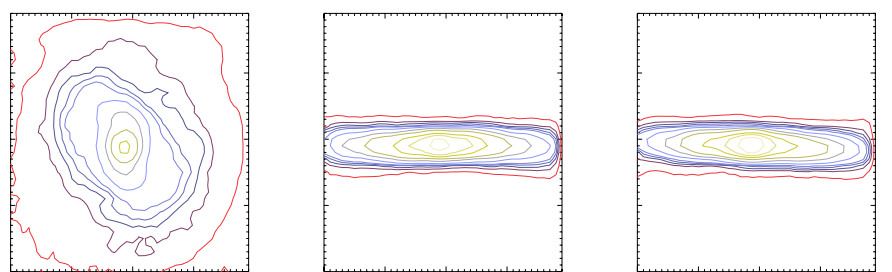

Fig. 11. Isodensity contours, in particular face-on, side-on and edgeon views of the inner bar of simulation $\mathrm{c} 7$ at $z=0$.

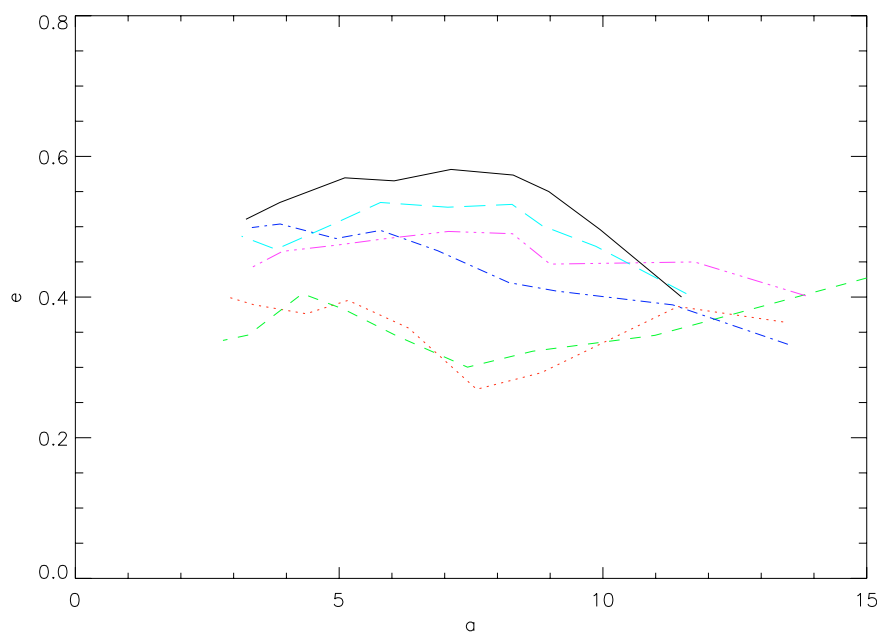

Fig. 12. Ellipticity as a function of the major axis, a (in physical kpc), of simulation $\mathrm{c} 1$ at different redshifts: $z=0$ continuous line, $z=0.25$ long-dashed line, $z=0.5$ long and short dashed line, $z=0.75$ dotdashed line, $z=1$ short dashed line, $z=1.25$ dotted line.

and c6 (Fig. 15). The cold cases end up with almost the same value of $Q_{\mathrm{t}}$ even if their evolution starts from different redshifts. The warmer case, instead, maintains a smaller value of the bar strength during all the evolution, in agreement with results obtained by using the ellipticity parameter. Table 3 shows that the final values (i.e. at $z=0$ ) of the bar strength evaluated with both these methods are consistent. According to the classification of Buta \& Block (2001), we assign class 1 to our less massive barred galaxies if their evolution starts from $z=2$ (i.e. simulations $\mathrm{c} 3$ and $\mathrm{c} 5$ ), class 2 if they evolve from $z=1$ (i.e. simulation c7), and class 4 to all the other ones (i.e. simulations $\mathrm{c} 1, \mathrm{c} 2, \mathrm{c} 4$ and $\mathrm{c} 6$ ). of the gravitational potential. We evaluated the components of the gravitational force on a suitable two dimensional grid using the method described by Buta \& Block (2001). However the information provided by such an approach could be affected by spiral arm torques and by some asymmetry in the bar itself (Buta \& Block 2001). Nevertheless, we succeeded in monitoring the behaviour of such a parameter for simulations c2, c4

\subsection{Comparisons with isolated cases}

In order to investigate the role of the cosmological framework on the bar instability, we perform isolated simulations using the same halo and the same disk-to-halo mass ratios as in our 


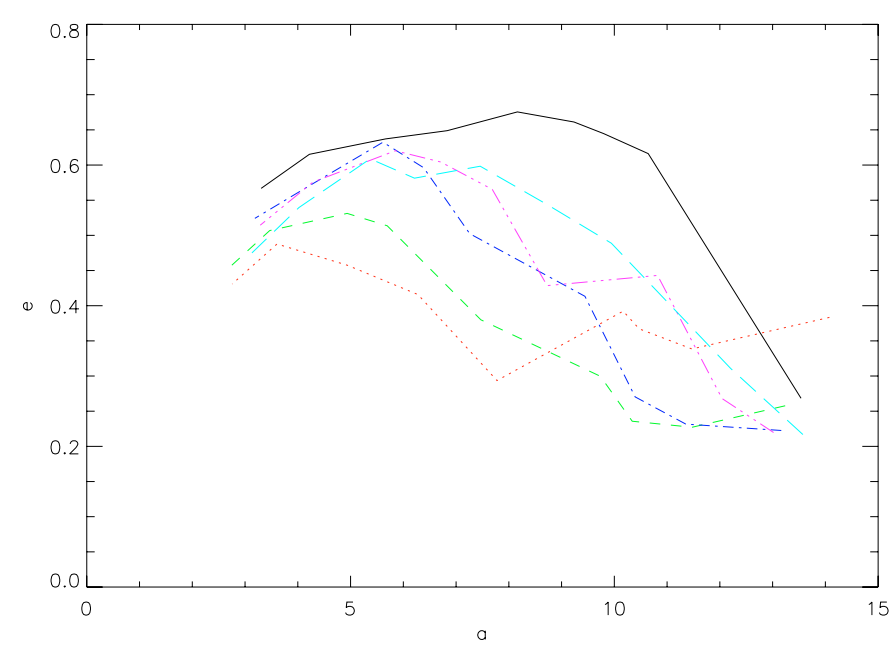

Fig. 13. Ellipticity as a function of major axis, a, of simulation c2 at different redshifts; symbols are as in Fig. 12.

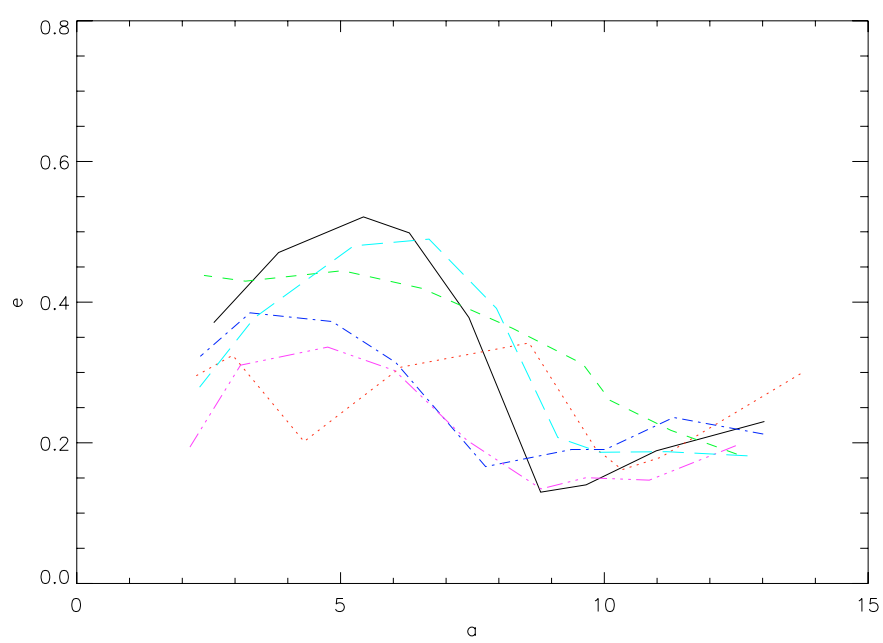

Fig. 14. Ellipticity as function of the major axis, a, for simulation $\mathrm{c} 4$ at different redshifts; symbols are as in Fig. 12.

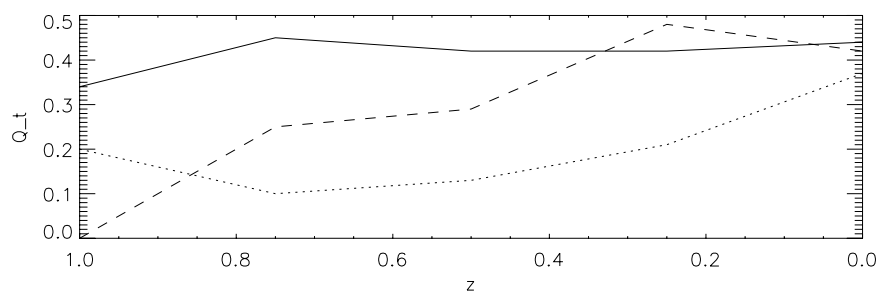

Fig. 15. Evolution of bar strength after $z=1$ evaluated using the gravitational torque (see text) for the simulations c2 (full line), c4 (dotted line), and c6 (dashed line).

cosmological setting (Sect. 4.2). We use $Q=1.5$ as the stability parameter in the disk. Our results show that the less massive disks do not show important differences as far as the bar feature is concerned: the bar strength is the same and the same bars in bar features arise.

In Fig. 16 we compare the halo radial density profiles of simulations c5 and i2. The density of the halo evolving in isolation becomes initially steeper, then it gradually flattens in the centre. In the outer regions, where the support of the

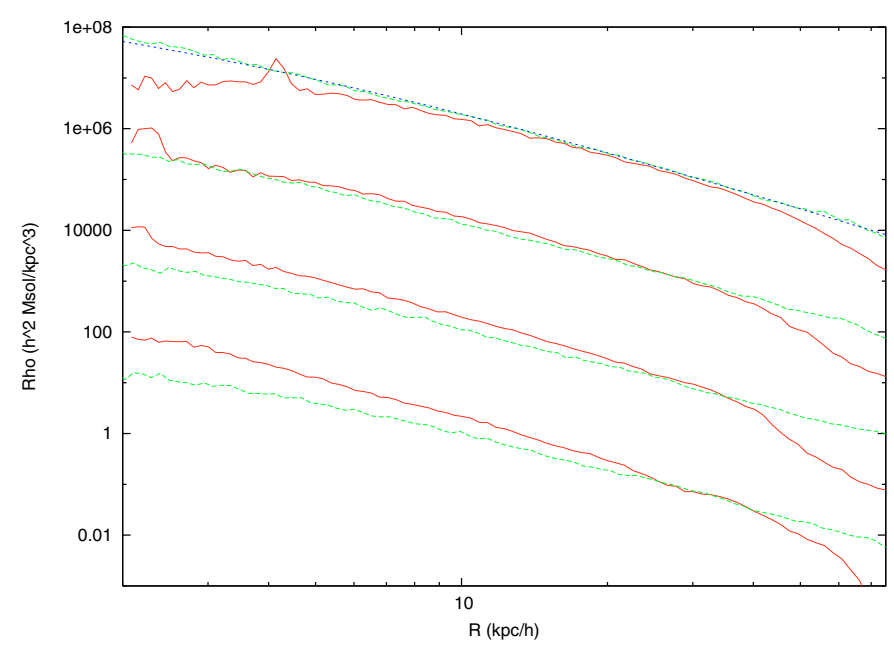

Fig. 16. Radial density profiles of the DM halo in simulations c5 (dashed lines) and i2 (solid lines) at redshifts $z=0,0.5,1.0,1.5$ from top to bottom for simulation $\mathrm{c} 5$ and at the equivalent evolutionary times for simulation i2. The three couples of profiles below have been divided by $10^{2}, 10^{4}, 10^{6}$ for clarity. We also show a NFW density profile having a concentration parameter $c \approx 23$ (dotted line), obtained as a two parameter best-fit of the density profile of simulation $c 5$ at $z=0$. Length units are in physical kpc.

cosmological environment is now lacking, the halo slowly loses matter toward bigger scales and the profile steadily steepens. On the other hand, the halo evolving in the cosmological environment continues to accrete mass and small substructures from larger scales. Such accretion is still significant up to redshift $z \approx 0.5$ at least (Fig. 1). Even if the dynamical evolution of the halo is different in cosmological and isolated simulations, the bar in the disk does form and evolves in a similar way. Thus we make the hypothesis that the common features of the two numerical experiments, namely the dynamical evolution and the anisotropy of the mass distribution, are the main engine for the bar instability. The large scale cosmological environment becomes a second order effect in the less massive disks. However the material accreting on the halo, which has been cut off with the halo segregation in a isolated system, plays a crucial role in the degree of the disk instability if the disk is not completely DM dominated. We conclude that the use of isolated halos in gravitational equilibrium for the study of the bar instability can give misleading results.

Taking into account our previous work in such an isolated non-cosmological framework (Curir \& Mazzei 1999; Mazzei $\&$ Curir 2001), we derive that live unrelaxed halos correspond to the most "realistic" approach available to simplify the picture. Even if the caveat outlined above cannot be forgotten, the dynamical state of the halo, as outlined in our work for the first time, plays a fundamental role in triggering and fuelling such an instability.

In order to disentangle the role of the halo's cosmological features like the prolate geometry and the spin on the instability, and to test the resolution effect, we produced an isolated halo with the same virial mass, radius and number of particles as our cosmological halo at $z=0$, but with an isotropic NFW radial density profile. The procedure is described by 

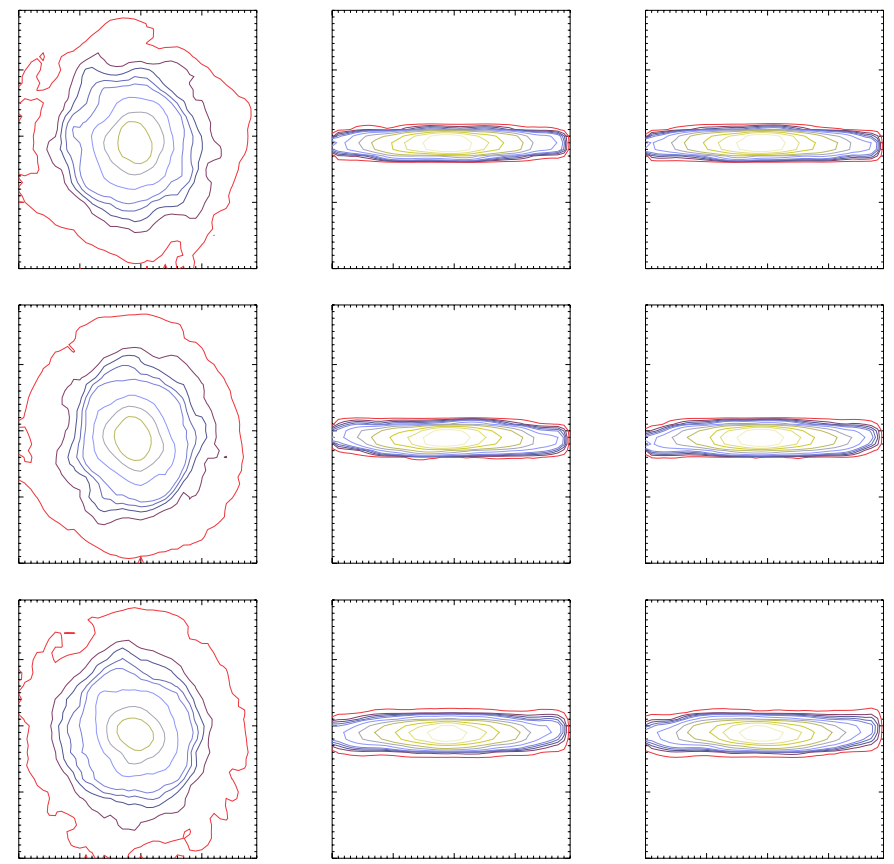

Fig. 17. Evolution of isodensity contours of the simulation i5 at different evolutionary times: from top to bottom, $t=5 \mathrm{Gyr}, t=7.5 \mathrm{Gyr}$ and $t=10 \mathrm{Gyr} ; x y, y z$ and $x z$ projections from left to right (see text (Sect. 7) for more details).

Hernquist (1993). We used a rejection technique to sample the density profile and we then assign a velocity to each particle following a local Maxwellian velocity dispersion. We checked that after 7 Gyr of evolution, the radial density profile of the halo is not changed, except for the "evaporation" of some particles dwelling in its outskirts. We then embedded a disk having the same mass, radius and $Q$ as in our simulation c5 and c3. These two simulations are labelled in Tables 2 and 3 as i5 and i4. According to the classical theory (Sect. 4.1), in simulation i5 the bar instability would be inhibited. We successfully reproduced this result with our live NFW halo (Fig. 17). Therefore the bar instability in simulation c5 is a genuine effect of the cosmological evolution and there is no evidence for a role of numerical noise. Moreover in simulation i5 the reaction of the DM halo to the disk immersion has not triggered a long-lived bar instability (see Fig. 17).

\section{Discussion}

In this work we investigate the issue of the bar instability in stellar exponential disks embedded in a DM halo selfconsistently evolving in a cosmological context. We aim to disentangle the effect of a few well-defined disk parameters on this instability. We also run isolated simulations using the same cosmological halo to analyse the effect of the whole cosmological framework on the results. This paper revisits in such a cosmological scenario the work by Curir \& Mazzei (1999). To compare our results with their paper we use the $R_{\mathrm{DM}}$ ratio in Table 2. Critical threshold values against $m=1$ and $m=2$ instability for this ratio have been calibrated by Athanassoula et al. (1987). They claim a value around 0.81 for the $R_{\mathrm{DM}}$ ratio to inhibit the lopsided instability (i.e. $m=1$ ) and around 2.2 to suppress the $m=2$ swing amplification instability. Even if these values are derived in a very simply framework, i.e. a isolated spherical and analytical DM halo, they are widely used in the literature (Bottema 2003; Elmegreen et al. 2003), therefore we will refer to this parameter to analyse our initial condition, in addition to the Efstathiou et al. parameter introduced in Sect. 4.1.

Looking at Table 2, simulations c1, c2, c4 and c6, which develop strong final bars (Table 3), are in the instability region for both these criteria. In particular in simulation $\mathrm{c} 1$, which also is below the threshold of lopsided instability (Athanassoula et al. 1987), the signatures of such an instability are clearly shown in the first phases of its evolution (Fig. 2). On the other hand simulations c3, c5 and c7 are stable according to both the criteria above. Nevertheless a weaker bar appears and lasts until the end of such simulations. Therefore the classical parameters are not good markers of the onset of the bar instability. In particular, when the self-gravity of the disk is negligible, i.e. the disk is DM dominated, the halo structure generated by the cosmology plays a crucial role in triggering such a instability.

Our findings agree with results of Mayer \& Wadsley (2004) in the isolated framework. They found that stellar systems with disk-to-halo mass ratios of 0.1 become bar unstable, regardless of the halo concentration and the $Q$ value, inside halos built up with suitable structural parameters derived from $\Lambda$-CDM cosmology, like their circular velocity at $R_{\text {vir }}, V_{\text {vir }}$, the NFW density profile and the spin parameter $(0.06$ and 0.1$)$. We point out that Mayer \& Wadsley (2004) do not take into account cosmological evolution for their halos. With the same disk-to-halo mass ratio Athanassoula (2002) found that such a instability is totally inhibited inside isotropic, non rotating halos with different density profiles (Eq. (1) of Athanassoula 2002), in agreement with the result of our simulation i5 (Sect. 7). This last result, together with those of simulations performed with different numbers of disk particles and with different softening lengths (Sect. 7) suggests that the development of long-living bars seen in our simulations is a genuine physical effect and not a numerical artifact.

Bar instability in the DM dominated cases is strongly affected by the halo models. Moreover structural details of the halo, related to the cosmological framework, drive morphological features of the stellar disk. Mayer \& Wadsley (2004) found a central bulge after $7 \mathrm{Gyr}$ which does not appear in our corresponding case. However such a bulge shows up in our intermediate self-gravitating case (simulation i1 in Table 2). This feature is also emphasised in the work by Athanassoula \& Misiriotis (2002) and Athanassoula (2003) for a disk-tohalo mass ratio of 0.2 , using the same halo presented in Athanassoula (2002) with the higher halo concentration.

Our results here are in good qualitative agreement with those by Curir \& Mazzei (1999) concerning their simulations 3, 4,7 and 8, with the same disk-to-halo mass ratio as in simulation $\mathrm{c} 1$ of Table 2, and also with their simulations 5 and 6 which correspond to a disk-to-halo mass ratio 0.2. However, their simulations 3 and 4 correspond to a relaxed halo, whereas 5, 6, 7 and 8 to a unrelaxed halo. In particular the $R_{\mathrm{DM}}$ initial values of their simulations 5 and 6 are respectively above and very near the 2.2 threshold value of bar instability, nevertheless the 
bar lasts until the end of their simulations $(\simeq 1.5 \mathrm{Gyr})$. Their simulations 1 and 2, which correspond to a relaxed dynamical state of a halo with disk-to-halo mass ratio 0.2, emphasise however a very different behaviour as far as the bar instability is concerned: the bar forms initially but degenerates then in a dense nucleus. Thus we argue that an unrelaxed dynamical state for isolated halo systems is more suitable to mimic a realistic "cosmological" halo, characterised by evolution, substructure and in-fall. This finding is important, since a vast majority of the work on the bar instability assumes a gravitationally stable halo.

\section{Conclusions}

In this work we present the first attempt to analyse the growth of bar instability in a fully consistent cosmological framework. We investigate such a issue in stellar disks embedded in a DM halo self-consistently evolving in a cosmological context. We aim to disentangle the effect of few well-defined disk parameters on this instability. We also run isolated simulations using the same cosmological halo to analyse the effect of the cosmological framework. Our results show that:

- stellar disks of different properties, i.e. mass and $Q$ parameter, embedded in the same halo and evolving in a fully consistent cosmological scenario, develop long living bars lasting up to redshift 0 .

- The classical criteria to account for bar instability cannot be validated in a cosmological framework where a bar always develops, due the halo evolution.

- The strength of the bar at $z=0$ is weakly dependent on the $Q$ parameter, for a given disk mass. However for the same disk-to-halo mass ratio, colder disks show stronger and longer bars. Thus the less massive warm disks entail the weakest bars, moreover a bars in bar is a common feature in their face-on morphology.

- Simulations performed embedding different disks in the same halo, extracted at $z=2$ from the cosmological framework, show that the effects of the large scale structures are negligible in the less massive, DM-dominated disks.

By comparing results in this work with our previous paper (Curir \& Mazzei 1999), we point out that live unrelaxed halos are the more suitable approach to mimic cosmological halos and to analyse bar instability in the less massive disks.

The mass anisotropy and the dynamical evolution of the DM halo have a crucial effect in enhancing and fuelling the bar instability, also in cases where ad hoc halo models provided stability predictions (e.g. Athanassoula 2003). The large-scale effects, such as the continuous matter infall on the halo and the infall of substructures during the whole time-span of the simulation, influence the bar strength and the details of its structure.

Acknowledgements. Simulations were performed on the CINECA IBM SP4 computer (Bo, Italy), thanks to the INAF-CINECA grants cnato43a/inato003 "Evolution of disk galaxies in cosmological contexts", and on the Linux PC Cluster of the Osservatorio Astronomico di Torino. We wish to thank for useful discussions: T. Abel, S. Bonometto, A. Burkert, E. D’Onghia, F. Governato, A. Klypin \& V. Springel.

\section{References}

Abadi, M. G., Navarro, J. F., Steinmetz, M., \& Eke, V. R. 2003, ApJ, 591,499

Athanassoula, E. 2002, in Disks of Galaxies: Kinematics, Dynamics and Perturbations, ASP Conf. Ser., 275, 141

Athanassoula, E. 2003, MNRAS, 341, 1179

Athanassoula, E., Bosma, A., \& Papaioannou, S. 1987, A\&A, 179, 23

Athanassoula, A., \& Misiriotis, A. 2002, MNRAS, 330, 35

Binney, J., \& Tremaine, S. 1987, Galactic Dynamics (Princeton, NJ:

Princeton Series in Astrophysics)

Bottema, R. 2003, MNRAS, 344, 358

Bullock, J. S., Dekel, A., Kolatt, T. S., et al. 2001, ApJ, 555, 240

Buta, R., \& Block, D. L. 2001, ApJ, 550, 243

Combes, F., \& Sanders, R. H. 1981, A\&A, 164, 173

Curir, A., \& Mazzei, P. 1999, A\&A, 352, 103

Debattista, V. P., \& Sellwood, J. A. 2000, ApJ, 543, 704

Efstathiou, G., Lake, G., \& Negroponte, J. 1982, MNRAS, 199, 1069

Elmegreen, B. G., Elmegreen, D. B., \& Leitner, S. M. 2003, ApJ, 590, 27

Ettori, S. 2003, MNRAS, 344, L13

Governato, F., Mayer, L., Wadsley, J., et al. 2004, ApJ, 607, 688

Hernquist, L. 1993, ApJS, 86, 389

Jesseit, R., Thorsten, N., \& Burkert, A. 2002, ApJ, 571, 89

Klypin, A., Kravtsov, A., Bullock, J., \& Primack, J. 2001, ApJ, 554, 903

Kravtsov, A. 1999, Ph.D. Thesis

Kravtsov, A., Klypin, A., \& Khokhlov, A. 1997, ApJ, 111, 73

Lacey, C. G., \& Ostriker, J. P. 1985, ApJ, 299, 633

Maller, A. H., Dekel, A., \& Somerville, R. 2002, MNRAS, 329, 423

Mayer, L., \& Wadsley, J. 2004, MNRAS, 347, 277

Mazzei, P. 2003, in Rec. Res. Devel. Astronomy and Astrophysics, 1, 457, Pandalai ed. (Kerala, India: Research Signpost)

Mazzei, P., \& Curir, A. 2001, A\&A, 372, 803

Mazzei, P., \& Curir, A. 2003, ApJ, 591, 784

Navarro, J. F., Frenk, C., \& White, S. D. M. 1996, ApJ, 462, 563

Navarro, J. F., Frenk, C., \& White, S. D. M. 1997, ApJ, 490, 493

Navarro, J. F., \& Steinmetz, M. 2000, ApJ, 538, 477

Patsis, P. A., \& Athanassoula, E. 2000, A\&A, 358, 45

Press, W. H., Flannery, B. P., Teuloski, S. A., et al. 1986, Numerical Recipes (Cambridge, UK: Cambridge University Press)

Power, C., Navarro, J. F., Jenkins, A., et al. 2003, MNRAS, 338, 14

Robertson, B., Yoshida, N., Springel, V., \& Hernquist, L. 2004, ApJ, 606,32

Sellwood, J. A. 1981, A\&A, 99, 362

Sellwood, J. A., \& Evans, N. W. 2001, ApJ, 546, 176

Springel, V., Yoshida, N., \& White, S. D. M. 2001, New Astronomy, 6,79

Stadel 2001, Ph.D. Thesis, see

http://www-hpcc. astro. washington.edu/tools

Vitvitska, M., Klypin, A. A., Kravtsov, A. V., et al. 2002, ApJ, 581, 799

Wechsler, R. H., Bullock, J. S., Primack, J. R., et al. 2002, ApJ, 568, 52 
A. Curir et al.: Bars in cosmology, Online Material p 1

\section{Online Material}


A. Curir et al.: Bars in cosmology, Online Material p 2
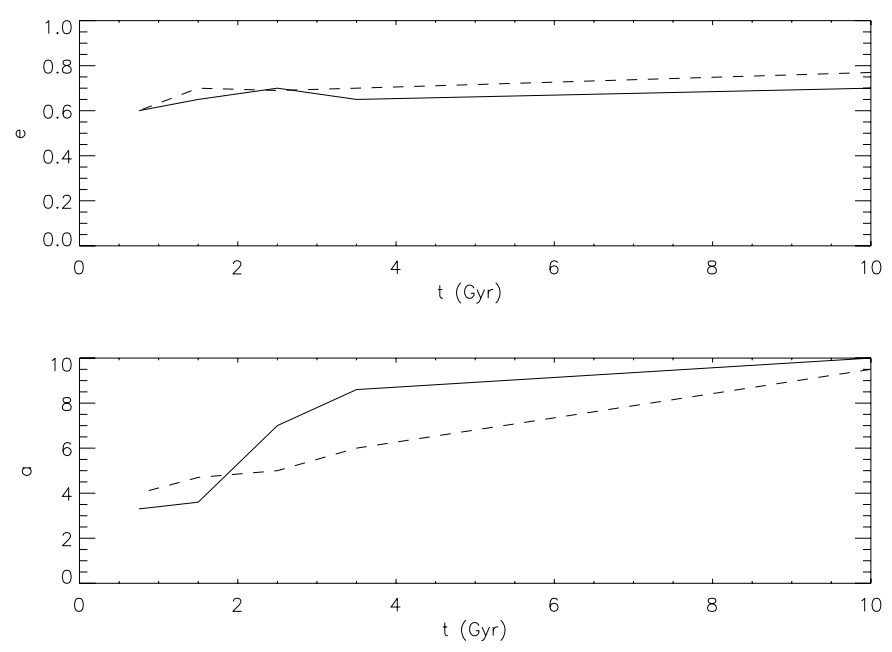

Fig. A.1. Evolution of the ellipticity (top panel) and of the bar length (bottom panel) of simulations i1 (dashed line) and i3 (full line).

\section{Appendix A: Numerical tests}

\section{A.1. Initialisation procedure}

While the disk is built to be in equilibrium with the gravitational potential of the halo (Sect. 2), the opposite is not true. This means that the halo contracts when we embed the disk, the amount of such a contraction depending on the mass of the disk. This effect roughly mimics the so-called "adiabatic contraction" (e.g. Jesseit et al. 2002) of a DM halo in a selfconsistent galaxy formation scenario, when the gas first collapses to form a disk. Nevertheless, in our approach the disk appears suddenly and also contracts in reaction to the augmented DM concentration. This double effect could trigger a bar instability. Therefore we modified the code to allow for the presence of a frozen stellar component. Its particles produce a gravitational force on the DM ones, but they are not subjected to any force. We built up an isolated frozen disk+halo system as described above (Sect. 4.2). Each particle of the disk is initially massless; such a mass increases linearly with time until a final disk-to-halo mass ratio of 0.3 . The system has been integrated in physical coordinates until $T=0.51 \mathrm{Gyr}$, which corresponds to $\approx 1.5 t_{\text {dyn }}$ (Sect. 4.2). After this period, a new disk having the same mass ratio is embedded, in equilibrium with the gravitational potential of the halo. We evolved this system up to the final time, $T=10.24 \mathrm{Gyr}$. This simulation is presented in Tables 2 and 3 as simulation i3. The presence of a very strong bar is seen during all the evolution. In Fig. A.1 we compare the evolution of the maximum bar ellipticity and of the corresponding semi-major axis length for simulation i3 and i1, in which the disk was added impulsively. We conclude that the reaction of the DM halo to the disk immersion, and the following counter-reaction of the disk to the increased halo concentration, are not the most important ingredient in producing the long-lasting bar we observe.
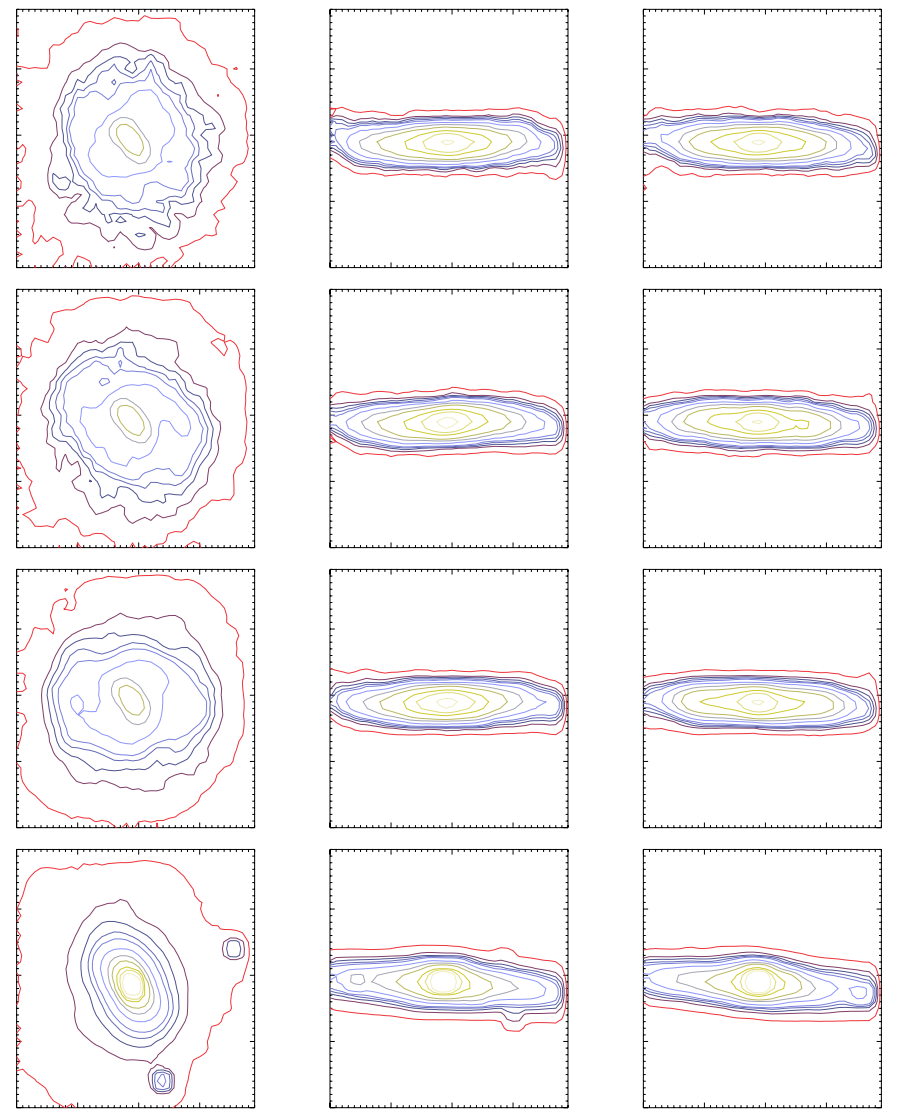

Fig. A.2. Isodensity contours at $T=7.0 \mathrm{Gyr}$ of isolated simulations i2 with different number of star particles, 9400, 18800,56000 and 560000 from top to bottom; $x y, y z$ and $x z$ projections are from left to right.

\section{A.2. The mass resolution}

With a fixed number of star particles, their mass varies by a factor of 10 in our simulations (Table 2). This can bring a different numerical scatter between star and DM particles. Problems connected with the resolution could be the occurrence of a bar instability triggered by numerical noise, which causes a poor sampling of the density field in the central part of the halo, and the numerical heating, which also depends on the number of particles involved (Lacey \& Ostriker 1985), could artificially weaken the bar instability. Therefore we run isolated tests with different number of star particles $(N=9400,18800,56000$, $560000)$. The results are slightly dependent on the mass resolution of disk particles (Fig. A.2). In particular the disk with 10 times more particles than our fiducial case keeps the bar feature, showing a more defined bar structure due to the higher resolution. We conclude that, even if there is a slight dependence of the details of the bar structure on the mass resolution of star particles, our main result, i.e. the occurrence of a long lasting bar instability even with this disk-to-halo mass ratio which would be classically stable, it is not caused by a resolution effect.

We are currently performing new cosmological simulations (as in Sect. 4.1) with higher mass resolution: a new halo consisting of 282134 particles at $z=2$ (compare with Table 1) and a stellar disk of 280000 particles. The disk-to-halo mass ratio, 
A. Curir et al.: Bars in cosmology, Online Material p 3
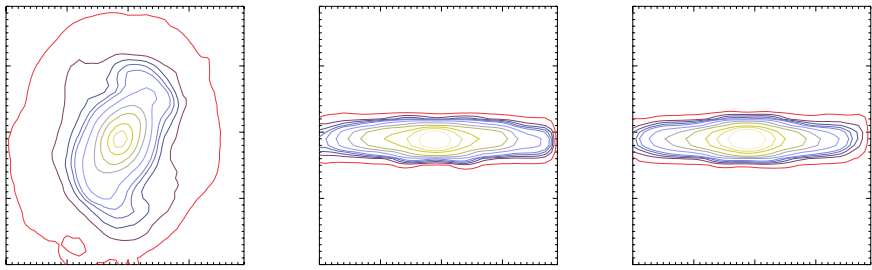

Fig. A.3. $x y, y z$ and $x z$ projections from left to right after $7 \mathrm{Gyr}$ for the high resolution cosmological run with disk-to-halo mass ratio 0.1 . See text (Sect. 7) for more details.

defined as in Sect. 4 , is $1 / 10$. The halo has a spin $\lambda=0.026$, a viral mass of $7.33 \times 10^{11} h^{-1} M_{\odot}$ at $z=0$ and a "quiet" mass accretion history, similar to that of the DM halo used in this work. Using such a halo, evolving in a different cosmological environment, we aim not only to improve the mass resolution of simulations in the cosmological framework but also to check how our results depend on the properties of the halo. Fig. A.3 shows that a bar is present for such a very light disk also with the higher resolution.

\section{A.3. The softening length}

Our fiducial softening is similar to the one suggested by Power et al. (2003) in their numerical convergence analysis of the radial density profiles of DM halos. Accounting for our number of particles, they suggest a minimum softening length: $\epsilon_{\min } \approx R_{200} / \sqrt{N}_{200}$, to prevent strong discreteness effects. From Table 1, this value corresponds to $\epsilon_{\min }=0.33 h^{-1} \mathrm{kpc}$ at $z=0$ and $\epsilon_{\min }=0.15 h^{-1} \mathrm{kpc}$ at $z=2$. Thus we run three simulations of the same isolated disk+halo system as in simulation i2 using three different softening lengths: $0.36 h^{-1}, 0.5 h^{-1}$ and $0.65 h^{-1} \mathrm{kpc}$. In Fig. A.4 we show the isodensity contours at times corresponding to redshifts $z=1$ and $z=0$ in the cosmological framework. The bar shapes and strengths are similar at $z=1$, where comparable inner bars (as given by two inner levels) are shown. We note the same similarities at $z=0$ with the exception of simulation with lower softening length, which shows more round inner contours. We conclude that, as far as the bar instability is concerned, varying the stellar softening within such range does not have a significant effect on the results.

\section{A.4. The disk radius}

The last simulation we performed explored weather the radius of the stellar disk can affect the triggering of bar instability. We therefore carried out a simulation with disk parameters as in simulation i1, but with both disk radius and scale length smaller by a factor of two. The results do not differ from the comparison case: the system shows a definite bar instability developing after $0.25 \mathrm{Gyr}$ and lasting until the end of the simulation.
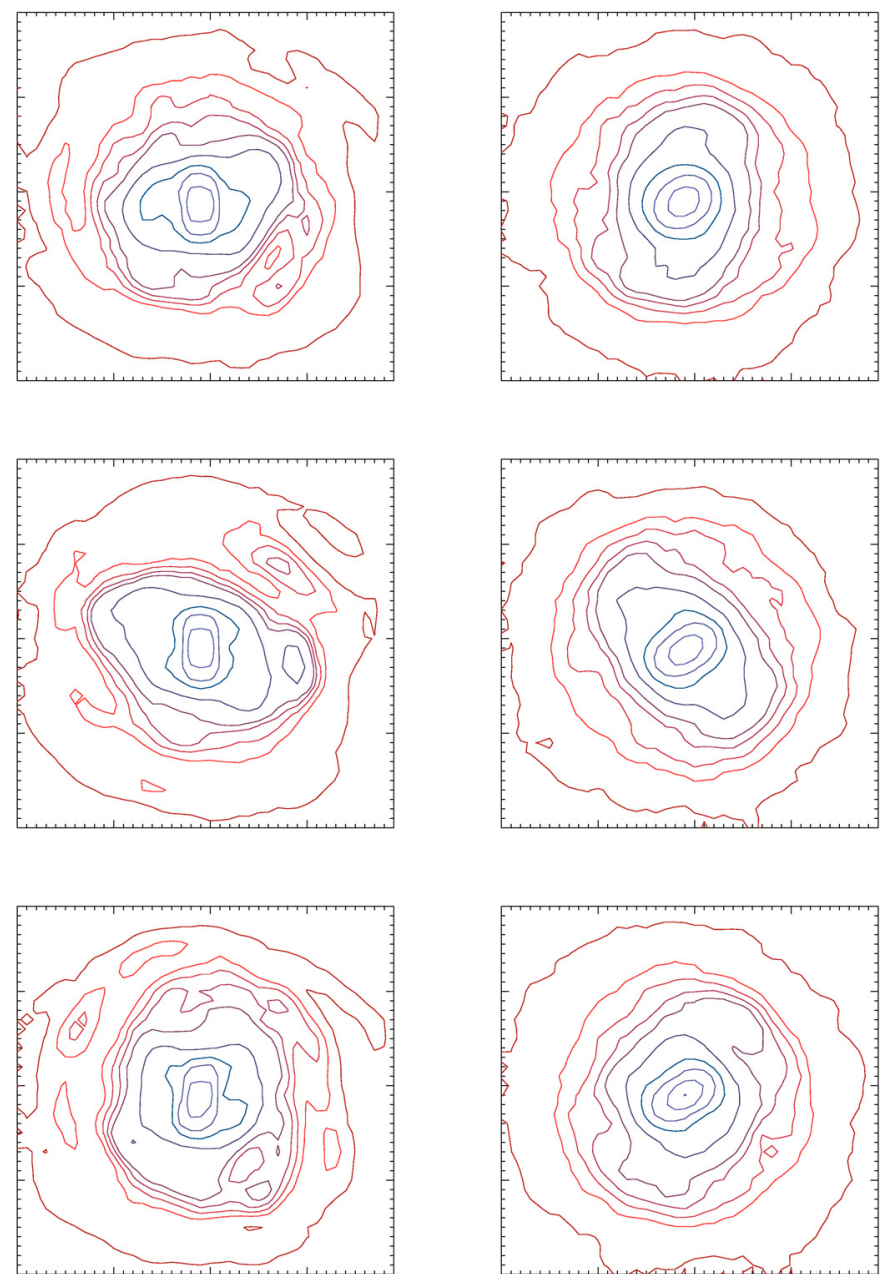

Fig. A.4. Face-on views at $T=7.71 \mathrm{Gyr}$ and $10.24 \mathrm{Gyr}$, corresponding to $z=1$ (left) and $z=0$ (right) in the cosmological framework, of isolated simulations i2 with stellar softening length $0.36 h^{-1}, 0.5 h^{-1}$ and $0.65 h^{-1} \mathrm{kpc}$ respectively from top to bottom (see text for more details). 